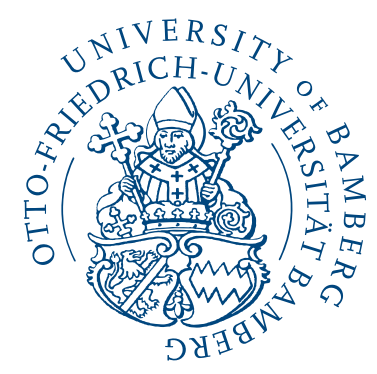

UNIVERSITY OF BAMBERG

\title{
Guidelines for Writing a High-Quality Thesis with the PSIThesis Template
}

\author{
BY \\ Dominik Herrmann \\ Privacy and Security in Information Systems Group \\ August 2020 \\ V20200807 \\ Links to this document: \\ https://doi.org/10.20378/irb-48428 \\ https://github.com/UBA-PSI/psi-thesis-guide
}





\section{Abstract}

For an overview of this document, see Chapter 1.

Provide a Thesis Abstract here (length: less than one page).

For a start, you may want to consult the concise instructions for writing the Summary Paragraph in Nature: https://www.nature.com/documents /nature-summary-paragraph.pdf. Moreover, consider Markus Kuhn's advice on differentiating abstract and introduction: https://www.lightbl uetouchpaper.org/2007/03/14/how-not-to-write-an-abstract/.

A boilerplate scheme for an abstract is as follows: devote $25 \%$ of the space on the purpose and importance of the research (introduction), $25 \%$ of the space on what you did (methods), $35 \%$ of the space on what you found (results), and $15 \%$ of the space on the implications of the research (cf. https://writingcenter.gmu.edu/guides/writing-an-abstract).

More concrete advice for writing abstracts can be found on the website of the Writing Center of the University of North Carolina at Chapel Hill (https://writingcenter.unc.edu/tips-and-tools/abstracts/). Some useful phrases for abstracts can be found at http://dissertation.laerd.com/usefu 1-phrases-when-writing-a-dissertation-abstract.php

Finally, you may also want to consider the excellent guide by Kent Beck on how to write good abstracts, which focuses on conference papers: https://plg.uwaterloo.ca/ migod/research/beckOOPSLA.html. 



\section{Contents}

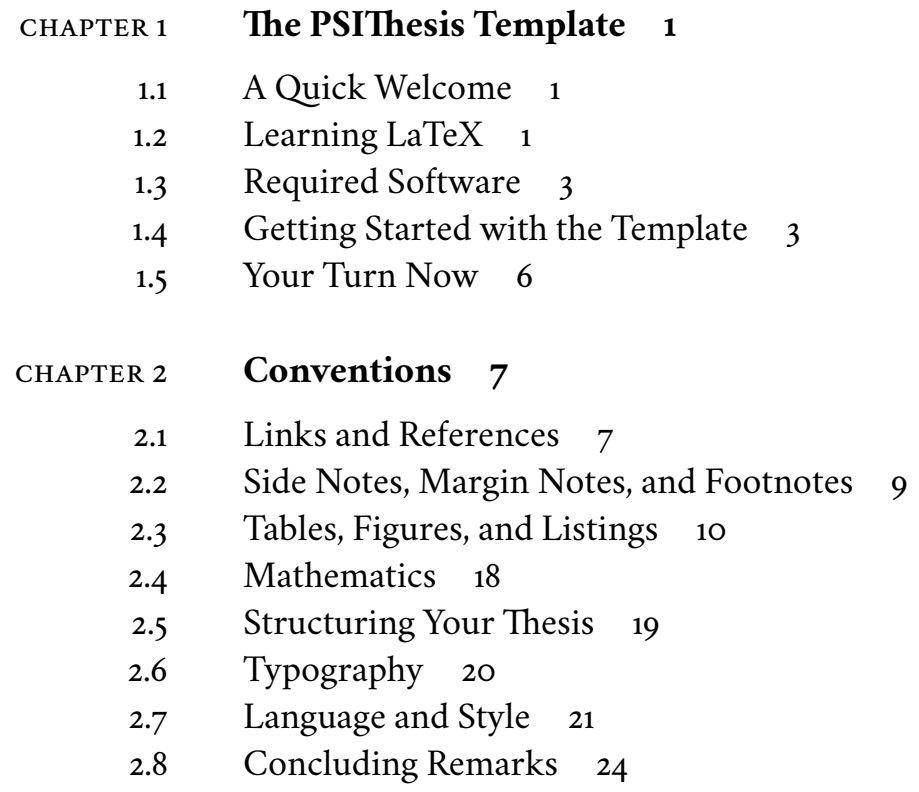





\section{The PSIThesis Template}

This introductory chapter will give you an overview of the PSI Thesis template and its usage. It also contains pointers to recommended reading for learning $\mathrm{BT}_{\mathrm{E}} \mathrm{X}$.

The remainder of this document presents conventions and recommendations that will help you create a coherent and visually appealing thesis. Chapter 2 explains the most critical aspects of scientific writing, including citations, URLs, tables, figures, typography, and layout.

Additional advice, which our students found useful in the past, follows in Appendix A. Advice on designing compelling figures and tables follows in Appendix B. Less-often needed details about the template, its history, and licensing details can be found in Appendix C.

\subsection{A Quick Welcome}

Welcome to this LaTeX thesis template guide. ${ }^{1}$ The PSIThesis template has been created mainly for students who want to submit a high-quality Bachelor's or Master's thesis to the Chair of Privacy and Security in Information Systems at the University of Bamberg (https://www.uni-bamberg.de/psi/). The template can also be used for seminar reports and $\mathrm{PhD}$ dissertations.

The PSITemplate and this thesis guide reflect, at least to some degree, the personal taste of members of the chair. We encourage our students to use the template without any changes. ${ }^{2}$

The template and the guide are available under an open license (cf. Sect. C.3). If you want to use the template for a thesis submitted at a different department or organization, feel free to make changes at your discretion. ${ }^{3}$

If you wish to contribute to the template or the guide, you may create an Issue or a Pull Request in the public GitHub repository at https://github. com/UBA-PSI/psi-thesis-guide.

\subsection{Learning LaTeX}

If you are new to LaTeX, we recommended to carry on reading this section.
1: It is based on the guide of the MastersDoctoralThesis template. The original text has been revised and extended. MastersDoctoralThesis is available at https://www.latextemplates.com/t emplate/masters-doctoral-thesis.

\footnotetext{
2: If you do have a different opinion on a particular aspect of the template or the guide, we will be happy to hear you out.

3: Redistribution of this guide and the template is subject to the details outlined in Sect. C.3.
} 
4: http://www.ctan.org/tex-archive/info/lshort/e nglish/lshort.pdf.

5: Find it under the "Additional Documentation" section towards the bottom of the page at http: //www.ams.org/tex/amslatex.html.

6: http://www.sunilpatel.co.uk/latex-type/latex -math-symbols/
If you are writing a thesis and its subject is technical, then creating it in LaTeX is highly recommended. LaTeX allows you to focus on the essential writing without having to worry over formatting or wasting time arguing with your word processor.

LaTeX can professionally typeset documents that run to hundreds or thousands of pages long. With simple mark-up commands, it automatically sets out the table of contents, margins, headers, and footers and keeps the formatting consistent and visually pleasing. One of its main strengths is the way it can easily typeset mathematics, even heavy mathematics.

LaTeX is not a wysiwyg (What You See is What You Get) tool, unlike word processors such as Microsoft Word or Apple's Pages. Instead, a document written for LaTeX is a simple, plain text file that contains no formatting. LaTeX is a "mark-up" language (like HTML): You tell the LaTeX processor about the desired formatting in simple commands amongst the text. For instance, if you want to use italic text for emphasis, you write the $\backslash$ emph $\{$ text $\}$ command and put the text you want in italics in between the curly braces.

\subsubsection{Introduction to $\mathrm{LaTeX}$}

If you are new to LaTeX, there is an excellent eBook, "The Not So Short Introduction to LaTeX" (aka "Ishort"), which is freely available online. ${ }^{4}$

To learn how LaTeX works, we recommend creating small test documents to reduce complexity. You can also learn from others by looking at other templates.

A Short Math Guide for LaTeX If you are writing a technical or mathematical thesis, you may want to read "A Short Math Guide for LaTeX". LaTeX supports many mathematical symbols, and it would take a great effort to memorize the commands for all of them. Sunil Patel's website shows the most common ones. ${ }^{6}$ You can use Sunil's page as a reference or crib sheet. The symbols are rendered as large, high-quality images, so you can quickly find the LaTeX command for the symbol you need.

\subsubsection{LaTeX Distributions}

The LaTeX distribution is available for Windows, Linux, and macOS. On Windows and Linux systems, the recommended distribution is TeX Live (https://www.tug.org/texlive/). The package for macOS is called MACTEX (http://www.tug.org/mactex/), and it contains all the applications you need - bundled together and pre-customized - for a fully working LaTeX environment and workflow. MACTEX includes a custom dedicated LaTeX editor called TEXShop for writing your '. tex' files and BiвDEsк, a program to manage your references and create your bibliography section. 


\subsection{Required Software}

To use the PSIThesis template, you need a working LaTeX installation with LuaLaTeX, biblatex, and biber. ${ }^{7}$ Usually, these tools are available in a typical LaTeX installation. We have tested the template with TeX Live 2019. We recommend to perform a full installation to ensure that all required LaTeX packages are available right away.

Use a current version of TeX Live that is available at https://www.tug .org/texlive/. Note that the TeX Live version packaged by major Linux distributions, such as Debian Linux, may contain an outdated version of lualatex.

Known Issues If you are using an outdated version of lualatex, compilation may fail with error: (vf): invalid DVI command (1). This is a known bug ${ }^{8}$ in old versions of lualatex that is triggered by the microtype package. In this case, we recommend upgrading to a current version of TeX Live.

Moreover, there is a known layout issue with old versions of the caption package. Version 3.4 of that package (released on 2019-09-11) is known to work well. ${ }^{9}$ You can update your TeX Live installation by running tlmgr.

Using Overlaf and XeTeX As of December 2019, the template does not work with https://www.overleaf.com. Overleaf uses the outdated version lualatex 1.07 from TeX Live 2018, which is subject to the bug mentioned above that prevents compilation of documents that use the microtype package.

The template includes microtype, not only because of the better typography but also because it uses microtype's command \textls\{text\} to change the letter spacing of the uppercase text on the title page.

You can use the template with Overleaf if you remove the line that loads the microtype package in setup. tex. Moreover, you will have to remove all calls to textls.

Another option is typesetting the template with XeTeX. To compile the template with XeTeX, you have to remove the line that loads the package luainputenc from setup.tex as well as all calls to textls.

\subsection{Getting Started with the Template}

Once you are familiar with LaTeX, you should explore the directory structure of the template (cf. Sections 1.4.1 and 1.4.2). Before you start to make changes, we recommend you to compile this guide on your machine (cf. Section 1.4.3). If there are no errors, it is time to place your details into the THESIS INFORMATION block of the main. tex file (cf. Section 1.4.4). You will also have to make some changes to the file misc/titlepage.tex, which sets up the title page.
7: We have compiled this guide with TeX Live 2019 (LuaLaTeX 1.10.0, biber 2.14), which was installed in January 2020.

8: https://de.comp.text.tex.narkive.com/fC1xfe b2/lualtex-microtype-error-vf-invalid-dvi-comm and-1

9: Check the version of caption in the log file that is created by LuaLaTeX during compilation.
Additional features of the template are described in Appendix C.1. 
10: The structure of a thesis may look like this:

- Chap. 1: Introduction

- Chap. 2: Background information

- Chap. 3: Experimental setup

- Chap. 4: Implementation considerations

- Chap. 5: Presentation of results

- Chap. 6: Discussion of results \& limitations

- Chap. 7: Conclusion and future directions

This chapter layout is specialized for an experimental thesis; your thesis may be different.

\subsubsection{Folder Structure}

This template comes as a single ZIP file that expands out to several files and folders. The folder names are mostly self-explanatory:

Appendices - this is the folder where you put the appendices. Each appendix should go into a separate .tex file. You have to include your appendix files in main. tex.

Chapters - this is the folder where you put the thesis chapters. Each chapter should go into a separate .tex file that is included from main. tex. ${ }^{10}$

Examples - this folder contains a Python script to generate a figure used in this guide. You do not need that folder for your thesis.

Figures - this folder contains all figures for the thesis. These are the final images that will go into the thesis document.

Two additional folders contain files that are internally used by the template. The folder fonts contains the TTF and OTF files of the template's fonts, the folder misc contains setup.tex, titlepage. tex, and the logo of University of Bamberg.

\subsubsection{Files}

Most of the template's files are plain text, and you can see their contents in a text editor. Important files are:

literature.bib - This is a BibTeX file that contains all the bibliographic information for literature that you cite in the thesis. You can write it manually, but there are reference manager programs (such as JabRef or BibDesk on macOS) that will create and manage it for you. Bibliographies in LaTeX are a subject of their own, and you may need to read about BibTeX before starting with this.

main.pdf - This is your typeset thesis created by LaTeX. It is part of the template's ZIP file. When you compile the template, you should get an identical version.

main.tex - This is the file that you tell LaTeX to compile to produce main.pdf. It contains the framework and constructs that tell LaTeX how to layout the thesis. It contains many comments that explain the purpose of each line of code. Fill in your details into the THESIS INFORMATION block.

titlepage.tex - This file creates the title page. In its original form, several elements (e. g., displaying your supervisor) are commented out because titlepage. tex sets up the title page of this document (i. e., the PSIThesis Guide). Please check the content next to the TODO markers and remove the comments as instructed.

PSIThesis.cls - This is the class file that tells LaTeX how to format the thesis. You should not have to make changes here. 
setup.tex - This file loads and sets up additional LaTeX packages. It controls the layout of the thesis. If you want to change the layout, you should do that here.

During compilation, LuaLaTeX and biber will create additional auxiliary files such as as main. aux, main.bbl, main.aux, main.blg, main. lof, main. log, main. lot, and main. out. The auxiliary files can be ignored or deleted. They will be regenerated as needed.

\subsubsection{Compiling the PDF}

You have to compile this template with lualatex (or XeTeX, cf. Sect. 1.3). Using pdfLaTeX is not possible, because the template uses TTF and OTF fonts.

On Windows, you can use the TeXworks application for compilation. To obtain the final PDF, you have to compile main. tex with lualatex, then run biber, and once more compile main. tex with lualatex.

On Linux and macOS, you can use the provided Makefile. ${ }^{11}$ Just navigate to the "en" directory and enter make in a terminal. Running make will automatically call the programs lualatex (which creates the PDF) and biber (which is used to compile the bibliography).

The make command keeps track of changes in your source files. If you add additional files that should be tracked for changes, you should edit the list of files at the top of the Makefile. Otherwise, make may refuse to compile a new version because it believes that main. pdf is already up to date. In this case, a call to make clean will help: It removes all files generated during compilation. After that, a call to make will regenerate them, including main.pdf.

We haven't prepared the template to be used with the convenient LaTeX editor LyX. ${ }^{12}$ LyX hides the LaTeX code from authors and offers a user interface that resembles a word processor. If LaTeX code puts you off, check out LyX and start writing there. Eventually, you can still export the LaTeX source code and copy and paste it into the PSIThesis template. Be sure to reserve some days to debug compatibility issues.

\subsubsection{Filling in Your Information in main.tex}

You will need to personalize the thesis template by filling in your details in main. tex with a text editor or your favorite LaTeX environment.

Open the file and scroll down to the third large block titled THESIS INFORMATION. You will see entries for University Name, Department Name, etc. Fill out the information about yourself, your group, and institution. ${ }^{13}$ You can also insert web links; if you do, make sure you use the full URL, including the http:// for this. If you don't want these to be linked, remove the $\backslash$ href $\{$ url $\}\{$ name $\}$ and only leave the name.

Next, open the file misc/titlepage.tex. Remove and add the comments as instructed by the TODO notes.
11: Alternatively, you should be able to compile the thesis by running latexmk -lualatex -pdf main.tex. If you use an IDE that does not support latexmk, you can still compile the document by manually executing lualatex, then biber, and lualatex once again.

12: https://www.lyx.org

13: If you write a thesis at the PSI Chair at the University of Bamberg, you can keep the defaults.

Do not forget to edit titlepage.tex! 
14: If you write a thesis at the PSI chair, your thesis should only contain a Table of Contents (i. e., neither a List of Figures nor a List of Tables). Therefore, all remaining lists are disabled by default. You must change main.tex, if you want to add these lists to your document.
When you have done this, save all changed files and recompile main.tex. All the information you filled in should now be in the PDF. You can now begin writing your thesis.

\subsubsection{More Information on main.tex}

The main. tex file sets up the structure of the thesis. There are plenty of comments that explain the purpose of the code. Each major document element is divided into commented blocks with titles in all capitals. Initially, there seems to be a lot of LaTeX code. Most of that code takes care of the formatting of the thesis, so don't worry about it.

Begin by checking that your information on the title page is correct. For the thesis declaration, your institution may insist on something different than the text given. If this is the case, replace the text in the DECLARATION PAGE block.

After that, you can insert a page with a quote (disabled by default). Next up is the abstract page, which concisely summarizes your work. After the abstract, you can insert an acknowledgments page (disabled by default). You can use this space to thank your supporters.

The table of contents and the list of figures and tables are taken care of for you. ${ }^{14}$ The next pages are optional: a list of abbreviations, a list of the physical constants and numbers, and a list of mathematical symbols. The next optional page contains a one-line dedication.

After the definitions of the lists, there is a block that includes all the individual chapters. Each chapter should be saved in a separate file and put into the chapters folder. Uncomment the respective lines (delete the \% character) as you add chapters to your thesis. Similarly for the appendices, uncomment the respective lines as you need them. Appendices should be saved in the appendices folder.

The next block sets up the bibliography. The template uses the bibliography style alpha. The alpha style creates reference labels that contain the first letters or initials of authors and a two-digit number for the year, such as [Hino3].

\subsection{Your Turn Now}

The easiest way to start your thesis is to replace text in the existing files. You might want to keep copies of the .tex to look up the source code as you move on.

We hope that this template helps you get up to speed. The tedious task of setting up the structure has been taken care of for you. It's now your job to create the content.

Good luck and happy writing! 


\section{Conventions}

An essential property of a long document, such as a thesis, is consistency. It is, however, challenging to keep track of terminology, style, and structure over an extended period.

Following certain conventions can help you achieve a consistent result. This chapter summarizes conventions and recommendations for effective thesis writing. Appendix A contains additional guidelines, while Appendix B provides more information on designing figures and tables.

This chapter contains only selected recommendations. For a more comprehensive list that also includes links to useful tools, consider https: //github.com/TheHairyJ/StudentResources/blob/master/Writing.md.

\subsection{Links and References}

You will most certainly include links and references in your thesis.

\subsubsection{Links to Websites}

Special care is necessary when you include links in your thesis. URLs are generally embedded using the $\backslash u r l\{\}$ command. Using this command allows LaTeX to break URLs at the end of a line. Moreover, the URLs will become clickable.

It is a frequently asked question whether one should embed all links as proper references (with a dedicated entry in the bibliography). We recommend to follow these principles: ${ }^{1}$

- Use a link in the text (or a footnote when it is a long link) when the primary purpose is to provide a reference to a particular location. Examples: websites of services or products or weblogs; when the aim is to make readers aware of its existence as a whole.

- Use a reference when the purpose is to refer to or discuss the content on a website. Note that bibliographic references should generally mention the author and the publication date (at least the year). If you cannot identify the author, you can resort to using the name of the respective organization instead (use an additional pair of curly braces in BibTeX - otherwise, LaTeX will misinterpret the name as first name and last name). Example: linking to a particular blog post.
Todo notes as this one can help with consistency. You can hide all todo notes before printing the final version by adding final to the documentclass.

Having a signpost paragraph before the first section in a chapter is considered good style. 
2: See also: Link Rot in Wikipedia.

3: We have archived all websites mentioned in this guide. In the interest of readability, we omit the archival remark after links.
Link Rot Many links become invalid at some point. Content may change or move to different URLs. Sites may go out of service altogether. This phenomenon is called link rot, and it has become an issue for academia. $^{2}$

Therefore, we ask you to archive all websites whose URLs you provide using The Wayback Machine of the Internet Archive, which is available at https://web.archive.org. Archiving preserves the content of a website at the given point in time and makes it permanently available in the Internet Archive.

It would be best if you informed the reader about the availability of an archived version. We recommend to provide the original URL and add "(archived at [date])" after the URL. ${ }^{3}$ Alternatively, you can just provide the archive.org URL of a location. This practice applies to URLs in the main text, in footnotes, and in references.

Archiving is not necessary for academic papers that have been published in a journal or a conference, for publications with a Digital Object Identifier (DOI) or an ISBN/ISSN as well as for papers that are available on a preprint server such as arxiv.org.

\subsubsection{Citation and References}

Whenever possible, you should cite the primary reference, i. e., the seminal paper that introduced a particular concept. When there is no fitting (seminal) paper, you can also choose to cite a well-known academic textbook that covers the topic. No citation is necessary for widespread knowledge, for instance, in the following sentence: "Many web sites rely on passwords for user authentication."

Most of the time, paraphrasing content is preferred over literally citing text with quotation marks. The most expressive way to integrate references to literature is to insert the labels right behind key terms or author names:

EMV systems [AM14] are used to secure payment cards. Murdoch et al. [Mur+10] have shown that EMV systems are broken.

Alternatively, you can append the label to the end of a sentence or sentence fragment. In this case, the label must come before the punctuation mark (such as a comma or period). An example is this sentence [Hino3].

When you paraphrase longer pieces, it is considered poor style to add the label at the end of a paragraph that paraphrases content from the literature. Instead, you should make this explicit at the beginning of a paragraph, e. g., like this:

The following paragraph describes the foo system [KAo3]....

This approach is also suitable to indicate more copious amounts of paraphrasing: 
Before we discuss the limitations of the foo system, we present its main functions in this section [KAo3]....

When you cite from books or long papers, you can insert the page number like this: \cite[p. 5] \{label\}, which results in [KAo3, p. 5].

When you cite multiple references in one spot, you should include them in one \cite command:

The FOO approach appears in a wide variety of applications [AM14; KAo3; Mur+10].

Note, however, that the result is not very informative. ${ }^{4}$ The following version provides much more information:

The FOO approach appears in a wide variety of applications, such as qux systems [Mur+10], baz systems [AM14], and much earlier - in bar readers [KAo3].

A remark on style: Do not treat references as nouns. You should, therefore, avoid "[Mur+10] shows that many EMV systems are broken ..." The label becomes less distracting like this: "Murdoch et al. show that EMV systems are broken [Mur+1o]."

BibTeX Snippets For many publications, you will find BibTeX snippets online, for instance, in the ACM Digital Library or on https://dblp. org. Using these snippets is generally recommended. Unfortunately, the quality of publicly available BibTeX snippets varies a lot. You will have to edit them to create a consistent and concise bibliography.

For instance, make sure that the name of a particular conference is always spelled consistently in all entries. Moreover, we encourage you to remove redundant information such as precise dates, multiple occurrences of the year, and the location of the conference. ${ }^{5}$ An example of a poorly styled reference is [Buc+o8], a cleaned-up item is [Die+18].

A common issue is the capitalization of words in titles. Whether you use sentence case or title case is up to you - as long as you stick to the choice. You may have to enclose the whole title of a bibitem in double curly braces to preserve capitalized letters. Alternatively, you can only enclose capitalized words or abbreviations in curly braces to protect them. ${ }^{6}$ Consider the following example, which ensures that TCP remains in uppercase:

@inproceedings $\{\ldots$, title $=$ "A new $\{$ TCP $\}$-based System", ... $\}$

\subsection{Side Notes, Margin Notes, and Footnotes}

The template offers you the option to use side notes, margin notes, and footnotes. We do not recommend footnotes ( $\backslash$ footnote) because the reader's eye has to travel long distances. You should use side notes (\sidenote) as a replacement instead of footnotes.
4: Source of this advice: https://nhigham.com /2014/12/22/more-tips-on-book-and-thesis-writ ing/

5: We have not cleaned up all items in the bibliography of this guide.

6: The template will print the items precisely as they are defined in the BibTeX file, i.e., you will probably not have to worry about abbreviations. 
7: This is a side note after a word.

8: Consider, e.g., the Chicago Manual of Style: "A note number should be placed at the end of a sentence or clause. The number follows any punctuation mark except the dash, which it precedes. It follows a closing parenthesis."
Due to the many examples and varying styles, this section is visually disturbing. Avoid such a wild mixture in your thesis.
You can also use margin notes (\marginnote). In contrast to side notes, margin notes are not numbered. By default, margin notes are set flush left or flush right (i. e., as close as possible to the main text), while side notes are justified.

Margin notes can be used to add remarks to a paragraph that are not closely related to a particular sentence or word. Moreover, you can use margin notes for links whose relationship with the main text is apparent. Finally, you can use margin notes for emphasis: placing essential keywords in the margin captures the reader's attention. Whatever you choose to use margin notes for, be consistent.

Like footnotes, side notes are either inserted directly after the particular word ${ }^{7}$ they refer to or after the punctuation if they relate to the whole clause or sentence. ${ }^{8}$ Side notes should be full, descriptive sentences (beginning with a capital letter and ending with a full stop).

Using a mixture of side notes, margin notes, and footnotes creates an inconsistent appearance and is therefore discouraged. If you decide to mix side notes and margin notes, consider changing the layout of margin notes to justified for better consistency. We have prepared commands for the necessary changes in setup.tex (look for the comment "justified margin notes").

One final remark on margins: If you cannot find any relevant content to put into the margin, leave it empty. We will use the space during grading for our comments.

\subsection{Tables, Figures, and Listings}

You should use tables, figures, and (optionally) listings to illustrate your writing. This section explains how these floating elements are included in the source code.

\subsubsection{Tables}

Tables are an important way of displaying your results in a concise way. Table 2.1 is an example of a table showing experimental results. This table was generated with the following LaTeX code:

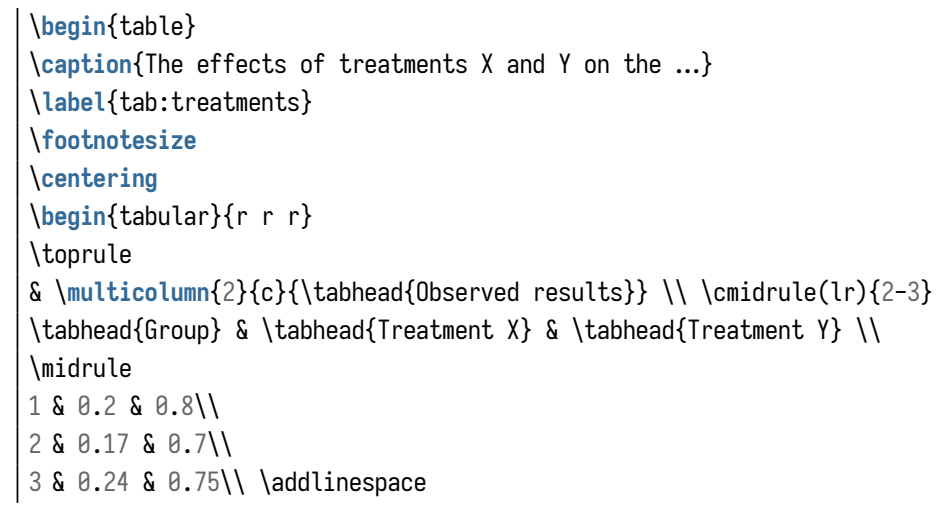


Table 2.1: The effects of treatments $X$ and $Y$ on the four groups studied.

\begin{tabular}{rrr}
\hline & \multicolumn{2}{c}{ Observed results } \\
\cline { 2 - 3 } Group & Treatment X & Treatment Y \\
\hline 1 & 0.2 & 0.8 \\
2 & 0.17 & 0.7 \\
3 & 0.24 & 0.75 \\
& 0.68 & 0.3 \\
4 & 0.61 & 0.9 \\
5 & 0.18 & 0.1 \\
6 & \\
\hline
\end{tabular}

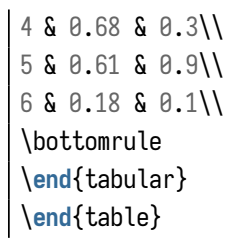

As shown in Table 2.1, column heads should use sentence-style capitalization, i. e., do not use title case as you would in section headings. In general, we recommend using a slightly smaller font size in tables than in the main text. The text in the example table uses footnotesize.

Tables have captions that always appear above the tabular environment. You must reference all tables in the main text at least once. To reference a table, you use the command $\backslash$ ref $\{<l a b e l>\}$, where $<$ label $>$ corresponds to a label defined within the table environment. See chapter2. tex for an example of the label and citation (e. g., Table 2.1).

In the PSI Thesis template, tables use the booktabs style, which avoids visual clutter such as vertical lines. If you want to group columns, do it in the headers using \cmidrule.

Horizontal lines should be used sparingly as well. Instead of horizontal lines, you should group consecutive rows with additional vertical space (using laddlinespace, cf. Table 2.1). You can also use bold print to highlight especially relevant parts.

Note that Table 2.1 is not laid out ideally: the numbers are given with different precision, which disturbs their alignment. Besides, strictly aligning all numeric columns to the right is not always appropriate. Centered alignment makes more sense here (cf. Table 2.2). For a step-by-step illustration of cleaning up tables, watch the presentation entitled "Less is more (attractive)". Moreover, consider the guidelines on organizing information in Sect. B.5 in the appendix.

Example of more complex tables are Tables 2.3 and 2.4. Table 2.4 shows how to build wide tables that span the margin and the text. If you need to create similar tables, feel free to re-use the code from chapter2.tex.

If you are in a hurry, you may find https://www.tablesgenerator.com and https://www.latex-tables.com useful. You can also use the convenient LaTeX editor LyX (https://www.lyx.org/) to build tables and export their source code.

https://www.darkhorseanalytics.com/blog/cle ar-off-the-table 
Table 2.2: The effects of treatments $\mathrm{X}$ and $\mathrm{Y}$ on the four groups studied.

\begin{tabular}{ccc}
\hline & \multicolumn{2}{c}{ Results } \\
\cline { 2 - 3 } Group & Treatment X & Treatment $\mathbf{~}$ \\
\hline 1 & 0.20 & 0.80 \\
2 & 0.17 & 0.70 \\
3 & 0.24 & 0.75 \\
& 0.68 & 0.30 \\
5 & 0.61 & 0.90 \\
6 & 0.18 & 0.10 \\
\hline
\end{tabular}

Table 2.3: A more complex table comparing experimentally obtained results for five systems.

\begin{tabular}{|c|c|c|c|c|c|c|c|c|c|c|c|c|}
\hline \multirow[b]{2}{*}{ OS } & \multirow[b]{2}{*}{ Tool } & \multicolumn{3}{|c|}{ A: Usability } & \multicolumn{4}{|c|}{ B: Security } & \multicolumn{4}{|c|}{ C: Privacy } \\
\hline & & A1 & $\mathrm{A} 2$ & A3 & A4 & B1 & $\mathrm{B} 2$ & B3 & $\mathrm{C} 1$ & $\mathrm{C} 2$ & $\mathrm{C} 2$ & $\mathrm{C} 4$ \\
\hline \multirow[t]{2}{*}{ Windows } & Bora & - & $\bullet$ & $\Delta$ & $\Delta$ & - & 口 & $\boldsymbol{\Delta}$ & $\Delta$ & $\Delta$ & $\Delta$ & $\square$ \\
\hline & Dicks & $\Delta$ & $\bullet$ & $\bullet$ & $\bullet$ & $\bullet$ & - & $\boldsymbol{\Delta}$ & $\square$ & $\square$ & - & $\Delta$ \\
\hline Android & NEW++ & $\boldsymbol{\Delta}$ & $\bullet$ & - & $\bullet$ & $\bullet$ & $\bullet$ & $\bullet$ & $\Delta$ & $\Delta$ & $\Delta$ & 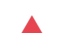 \\
\hline macOs & P2I & $\Delta$ & $\Delta$ & - & 口 & - & • & $\Delta$ & \multicolumn{4}{|c|}{$\ldots$... no results } \\
\hline $\mathrm{iOS}$ & Carmadillo & $\Delta$ & $\Delta$ & - & $\bullet$ & 口 & $\Delta$ & $\Delta$ & $\Delta$ & 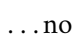 & resi & \\
\hline
\end{tabular}

Further Reading Creating good tables is a challenge on its own. Recommended readings:

- Nick Higham. Better LaTeX Tables with Booktabs (https://nhigham.c om/2019/11/19/better-latex-tables-with-booktabs/),

- Lapo Filippo Mori. Tables in $\mathrm{ET}_{\mathrm{E}} \mathrm{X} 2 \varepsilon$ : Packages and Methods (http: //tug.org/pracjourn/2007-1/mori/mori.pdf),

- Markus Püschel. Small Guide to Making Nice Table (https://inf.ethz. $\mathrm{ch} /$ personal/markusp/teaching/guides/guide-tables.pdf), and

- Adrian P. Robson. ${ }^{\mathrm{ET}} \mathrm{E} \mathrm{X}$ Table Hints and Tips (https://www.cl.uni-he idelberg.de/courses/ss19/wissschreib/material/tableTricks.pdf).

- Appendix B.5 in Appendix B offers some high-level advice on how to organize data for its effective presentation in tables.

\subsubsection{Figures}

This section provides general advice and pointers on designing compelling figures. After that, it explains the layout options offered by this template. Appendix B contains guidance on how to design figures on a conceptual level.

\subsubsection{Effective Figure Design}

There will hopefully be many figures in your thesis. Creating effective and visually pleasing figures is challenging. 
Table 2.4: Overview of related literature on the topic of the thesis.

\begin{tabular}{|c|c|c|c|c|c|}
\hline Class & No. & Description & Authors & Year & Relevant \\
\hline \multirow[t]{3}{*}{ A } & 1 & $\begin{array}{l}\text { Northern Wheatears had higher reproductive success in } \\
\text { higher vegetation height }\end{array}$ & Arlt and Part & 2007 & 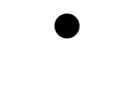 \\
\hline & 2 & $\begin{array}{l}\text { Mallards that avoided wetlands with large expanses of open } \\
\text { water had higher reproductive success }\end{array}$ & Bloom et al. & 2013 & \\
\hline & 3 & $\begin{array}{l}\text { American Redstarts that occupied wet forest habitat in the } \\
\text { winter raised more offspring than conspecifics occupying } \\
\text { other habitats in the winter }\end{array}$ & Norris et al. & 2003 & ( ) \\
\hline \multirow[t]{3}{*}{$\mathrm{B}$} & 4 & $\begin{array}{l}\text { Great Tits nesting in mature woodland produced larger } \\
\text { broods than conspecifics nesting in gardens and hedgerows }\end{array}$ & Riddington and Gosler & 1995 & \\
\hline & 5 & $\begin{array}{l}\text { Reduction in winter stubble has led to a reduction in Reed } \\
\text { Bunting survival rate }\end{array}$ & Peachet al. & 1999 & 0 \\
\hline & 6 & $\begin{array}{l}\text { Fledgling Ovenbird survival increased with vegetation struc- } \\
\text { ture }\end{array}$ & King et al. & 2006 & 0 \\
\hline $\mathrm{C}$ & 7 & $\begin{array}{l}\text { Daily nest survival of eight forest species was positively re- } \\
\text { lated to nest distance from the forest edge and nest height }\end{array}$ & Newmark et al. & 2011 & \\
\hline
\end{tabular}
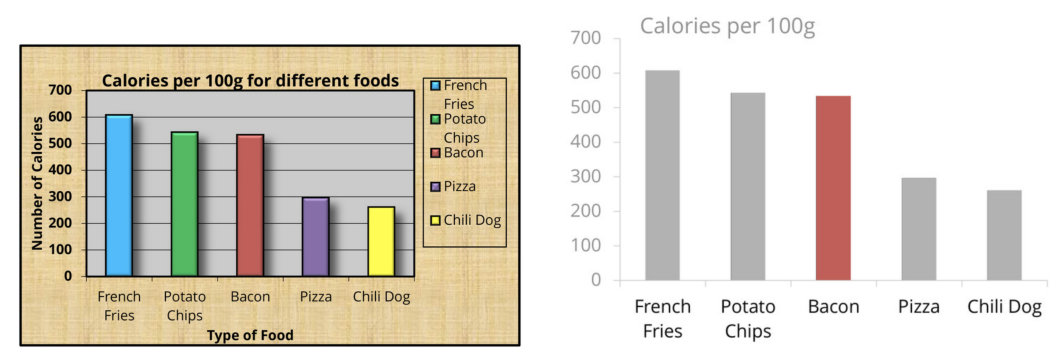

Ideally, you draw all figures yourself to ensure that they use a consistent graphical language, including colors, fonts, text sizes, line styles, arrowheads, fill styles, padding, margins, and the radius of rounded corners. Ensure to align elements properly and to provide sufficient whitespace.

When it comes to figures and data plots, less is more! According to Edward Tufte, ${ }^{9}$ you should remove all non-data ink and redundant data ink, within reason, to increase the data-to-ink ratio and create a sound graphical design. In particular, you should avoid shadows and unnecessary ${ }_{3} \mathrm{D}$ effects.

Watch the illustrative presentation entitled "Remove to improve." The plots in Fig. 2.1 (taken from linked website) demonstrate the benefits of removing unnecessary clutter. Find more guidelines in Appendix B.

Ensure that your figures work when printed in monochrome, i. e., you shouldn't rely on the colors too much. If you do use colors, use the same color palette in all figures, and ensure that readers with color blindness can differentiate all colors.

If you present plots of experimental results, use a tool that allows you to recreate plots automatically. To this end, we recommend to writing scripts that create the plots based on raw data. Another benefit is that you will be able to change the design of all plots with little effort. Common
Figure 2.1: Removing unnecessary clutter.

9: Edward Tufte (1983): The Visual Display of Quantitative Information.

https://www.darkhorseanalytics.com/blog/dat a-looks-better-naked/

https://matplotlib.org, https://seaborn.pyd ata.org, https://ggplot2.tidyverse.org, and http://www.gnuplot.info 


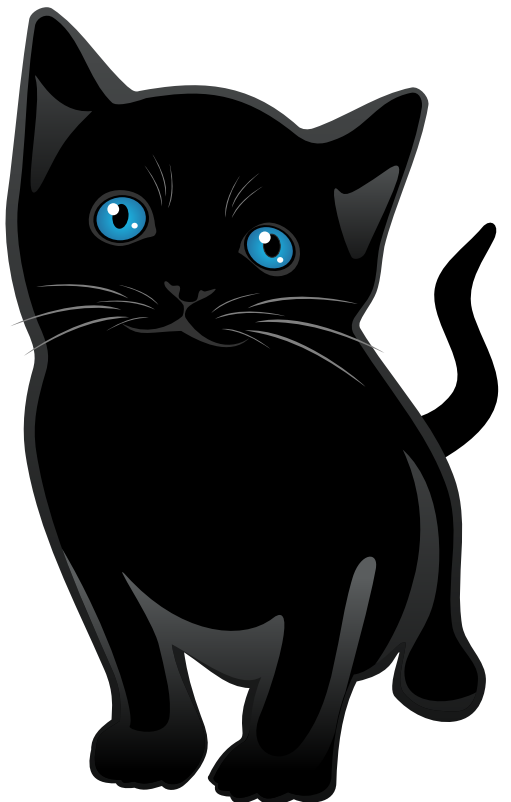

Figure 2.2: A black cat (Source: https://www.stockvault.net/photo/147894/lit tle-cat-vector ("non-commercial license")).

tools are Python's matPlotlib (consider using SEABORN), R's GGPLOT2, and GNUPLOT. Export your plots as PDFs for high quality.

\subsubsection{Figure Layout Options}

Place your figures in the figures folder, which allows you to omit the directory name in the \includegraphics \{filename $\}$ command. The following code produces Fig. 2.2:

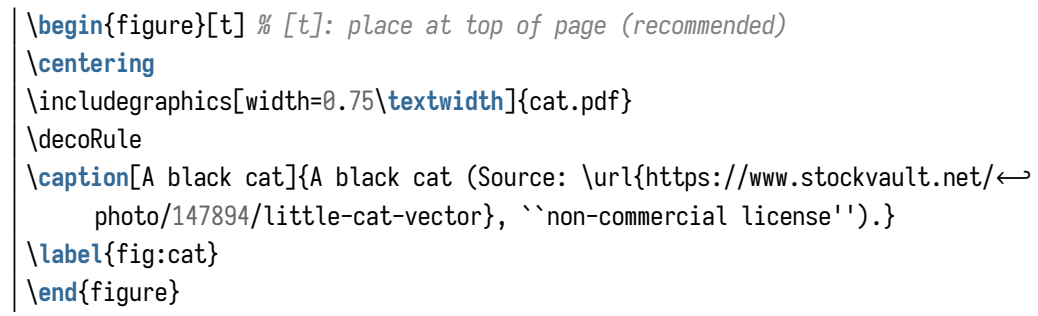

You can also use the custom command image for inserting a figure:

1 |image[h]\{ $\backslash$ textwidth $\}\{$ A black cat $\}\{$ An image of a black cat. $\}$ cat.pdf $\}$

This command will produce the same result as the previously shown commands. For further information on the usage of the custom commands, you should take a look at the extensive documentation in commands. tex in the misc folder.

Figures should appear on the page where they are referenced first or on one of the subsequent pages. The recommended figure placement is the top of the page (denoted by [t]). Don't worry about figures not appearing exactly where you write them in the source. Sometimes there 
is not enough room to fit a figure directly where it should go (in relation to the text) and so LaTeX puts it at the top of the next page.

Every figure needs a descriptive caption and a label. Figure captions must always appear below the included graphics file within the figure environment.

Every figure must be referenced in the text at least once, either in parentheses (cf. Fig. 2.2) or explicitly in the sentence: Figure 2.2 shows an electron. Refer to figures using the abbreviation Fig. followed by a protected space $(\sim)$ and \ref. Exception: write Figure if it is the first word of a sentence. Note Fig. and Figure are capitalized when they are used as part of a reference.

The \caption command contains two parts, the first part, inside the square brackets $^{10}$ is the title that will appear in the List of Figures, and so should be short. The second part in the curly brackets should contain the longer and more descriptive caption text.

The \decoRule command is optional and simply puts a horizontal line below the image. Such a line can be useful with figures that are not symmetrical or whose exterior is uneven at the bottom. There is also the more flexible variant $\backslash$ decoRuleFlex $\{\mathrm{dim}\}$, where dim determines the length of the rule, e. g., 1.0\textwidth.

Resize your figures, ideally consistently, to an appropriate size, e. g., a fraction of $\backslash$ textwidth. Check that the font size (after scaling) is consistent. This advice is especially important when you include figures with different aspect ratios.

LaTeX is capable of using images in PDF, JPG, and PNG format. Whenever possible, use vectorized figures (PDF). PDFs provide sharper results at smaller file sizes. If you have to use pixel graphics, create them with a sufficiently high resolution (at least $300 \mathrm{dpi}$ ).

Wide Figures You can also add wide figures that span the full width of a page. Wide figures should be placed at the top of the page. The following code creates the example figure (Fig. 2.3):

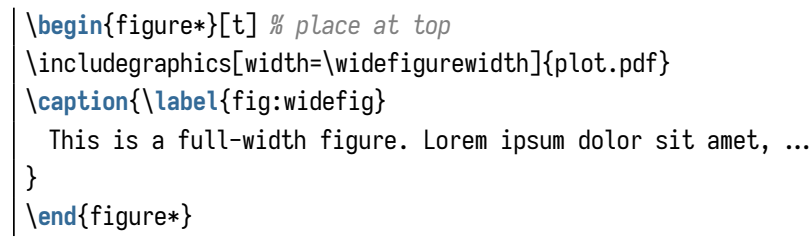

You can also use the custom command wideimage for inserting a figure:

1 |wideimage[h]\{plot.pdf\}\{This is a full-width figure. Lorem ipsum dolor sit $\hookleftarrow$ amet, ...\}\{widefig\}

This command will produce the same result as the previously shown commands. For further information on the usage of the custom commands, you should take a look at the extensive documentation in commands.tex in the misc folder.
10: Theses at the PSI Chair usually do not have a List of Figures. You can, therefore, omit the part in square brackets. 


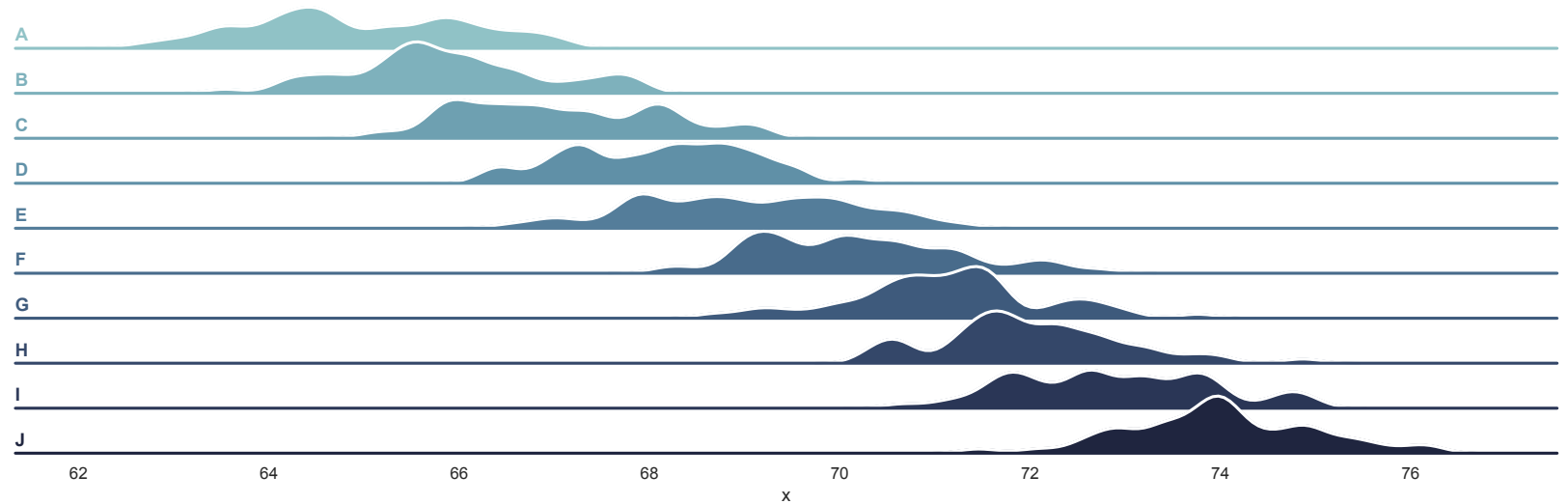

Figure 2.3: This ridge plot is an example of a full-width figure (Source: plot.py in the examples folder, obtained from https://seaborn.pydata.org/examples/kde_ridgeplot.html). Lorem ipsum dolor sit amet, consectetur adipisicing elit, sed do eiusmod tempor incididunt ut labore et dolore magna aliqua.

Occasionally, LaTeX misplaces wide figures on the horizontal axis. For instance, figures may end up partly outside the page instead of properly aligned with the text block. Often, these problems happen only sporadically. Compiling main. tex again with a single invocation of lualatex will fix the layout.

Side-by-Side Figures Sometimes, it is desirable to show multiple figures next to each other. There are several approaches for that, e. g., using the subfigure package. In many cases, however, comprehensive subfigure support is not needed. A lightweight approach, as shown in Fig. 2.1, may be sufficient. This result is achieved with the following code:

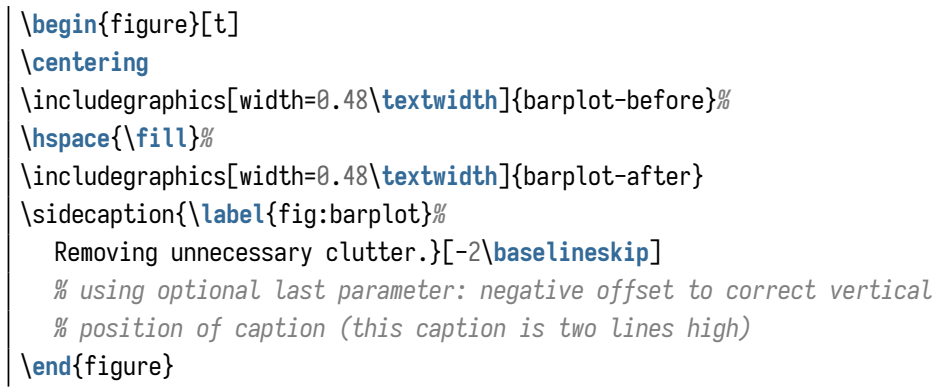

You can also use the custom command twoimages for inserting two figures next to each other:

1 Itwoimages[h] barplot-before $\}$ barplot-after $\}\{$ Removing unnecessary clutter. $\}\{\hookleftarrow$ barplot\}

This command will produce the same result as the previously shown commands. For further information on the usage of the custom commands, you should take a look at the extensive documentation in commands. tex in the misc folder.

Figure 2.1 uses a side caption (sidecaption). Whether you use side captions or not is up to you. Just ensure to place all captions consistently, 
i. e., either in the margin or above or below floats for tables and figures, respectively. Note that sidecaption does not work with floating listings.

Margin Figures Sometimes, it makes sense to place figures in the margin. Margin figures work best for figures that are simple and unobtrusive. They are a useful mechanism to include figures that illustrate concepts and graphs that are meant to give an overall impression. Do not use margin figures for figures that convey essential results.

You can create margin figures like Fig. 2.4 with the following code:

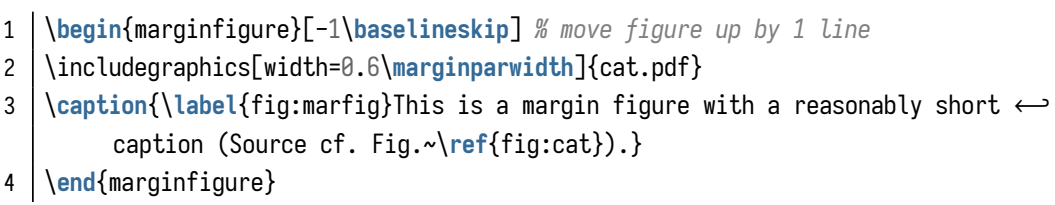

You can also use the custom command marginimage for inserting a figure in the margin:

1 |marginimage[-1]\{cat.pdf\}\{This is a margin figure with a short caption. $\}\{$ marfig\}

This command will produce the same result as the previously shown commands. For further information on the usage of the custom commands, you should take a look at the extensive documentation in commands.tex in the misc folder.

Margin Tables You can also place tables into the margin. Due to the limited space of the margin, the margin table's content should be carefully considered. Otherwise, the table will clip into the main body or overflow over the page. In general, we recommend to create the table with the full width of the margin. Otherwise, the margin layout may become ragged. Moreover, we reduce the font size of margin tables to footnotesize so that they do not attract too much attention. You can create a full-width margin table like Table 2.5 with the following code:

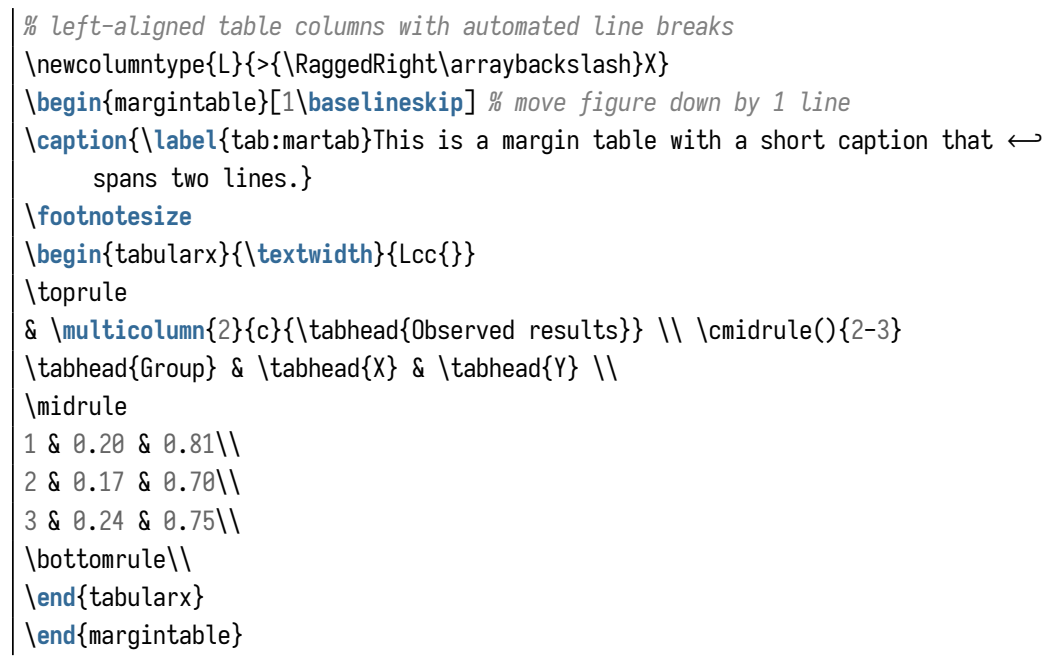

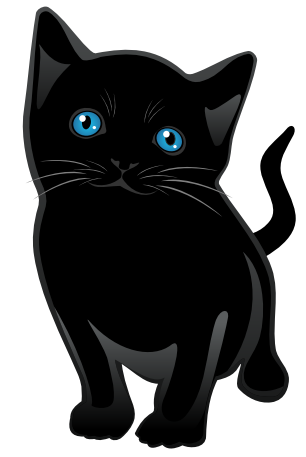

Figure 2.4: This is a margin figure with a reasonably short caption (Source cf. Fig. 2.2).

Table 2.5: This is a margin table with a short caption that spans two lines.

\begin{tabular}{lcc}
\hline & \multicolumn{2}{c}{ Observed results } \\
\cline { 2 - 3 } Group & $\mathbf{X}$ & $\mathbf{Y}$ \\
\hline 1 & 0.20 & 0.81 \\
2 & 0.17 & 0.70 \\
3 & 0.24 & 0.75 \\
\hline
\end{tabular}


11: If you have Python, you can consider the more modern package minted, which uses pygments for syntax highlighting.
12: As of now, the template does not offer wide listings that span the text and the margin.

\subsubsection{Listings}

If you are new to LaTeX, we recommend using the listings package for code listings. ${ }^{11}$ With the template's monospace font, lines can have about 85 characters. Longer lines are automatically wrapped. An arrow at the end of the line indicates the line-wrapping. Format your listing in such a way that the number of wrapped lines is low to improve readability.

You can create floating listings, which behave very much like floating figures and tables, i. e., they have a caption and can be referenced (cf. Listing 2.1 for an example). ${ }^{12}$ Sometimes, however, the properties of floating listings are undesirable. For short code fragments, it often makes more sense to use a non-floating listing.

Listing 2.1 has been generated with the following code:

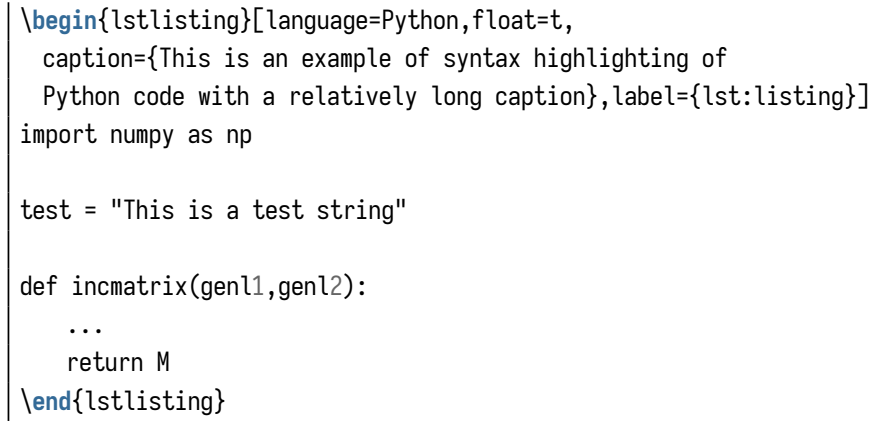

\subsection{Mathematics}

The "Not So Short Introduction to LaTeX"13 should tell you everything you need to know for most cases of typesetting mathematics. If you need more information, a much more thorough mathematical guide is available from the AMS. ${ }^{14}$

There are many different LaTeX symbols to remember, luckily you can find the most common symbols in "The Comprehensive ETEX Symbol List” (http://ctan.org/pkg/comprehensive).

You can write a numbered equation like this:

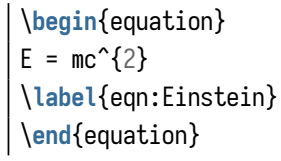

This will produce Einstein's famous energy-matter equivalence equation:

$$
E=m c^{2}
$$

LaTeX automatically gives all equations you write equation numbers. If you don't want a particular equation numbered, use the unnumbered form: 
Listing 2.1: This is an example of syntax highlighting of Python code with a relatively long caption

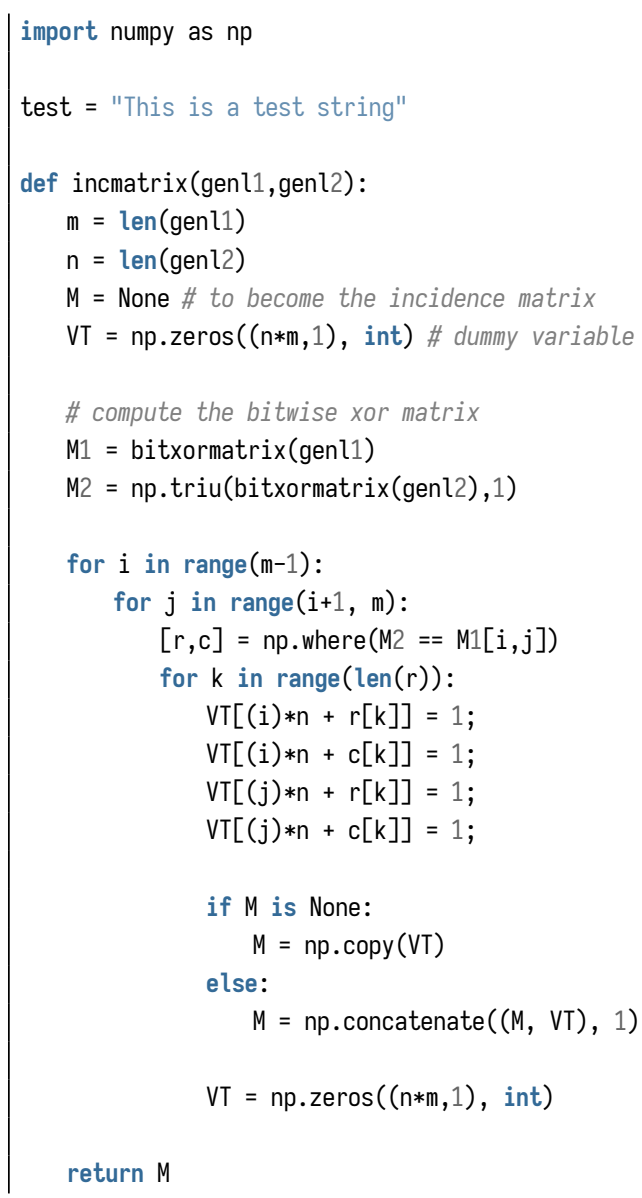

You can also have equations in the middle of a paragraph, e.g., $x=$ $\sum_{i=1}^{10} \cdot \hat{a} \cdot \frac{\alpha}{\psi} \cdot i^{2^{3}}$, with this syntax:

$1 \mid$ e. $\backslash, g ., \backslash\left(x=\backslash\right.$ sum_ $\{i=1\}^{\wedge}\{10\} \backslash$ hat $\{a\} \backslash \operatorname{codot} \backslash$ frac $\{\backslash$ alpha $\}\{\backslash p s i\} \backslash$ cdot $i^{\wedge}\left\{2^{\wedge} 3\right\} \hookleftarrow$ I), with this syntax:

\subsection{Structuring Your Thesis}

You should break your thesis up into chapters and sections. LaTeX automatically builds a Table of Contents by looking at all the Ichapter\{\}, Isection \{\} and \subsection \{\} commands you write in the source. You may even think about using subsubsections (\subsubsection\{\}). All of these will be hierarchically numbered.

Use (sub-)subsections only if you use them consistently in all or at least multiple chapters. Otherwise, you should opt for a paragraph\{\}, which structures pieces of content with bold non-numbered inline headings (examples throughout this guide). 
15: To allow for fast skimming, we use bold print liberally throughout this guide (which would not be appropriate in a thesis).
16: More on the topic "Italics or Scare Quotes?" is available at https://sblhs2.com/2016/09/15/i talics-scare-quotes/.

17: Reasons given in "Quotes When Nothing Is Being Quoted", which is available at https://style .mla.org/quotes-when-nothing-is-being-quoted/.
Avoid dangling elements in the hierarchy: If you have a Section 1.1, you should also have (at least) Section 1.2. This rule applies to all levels of the hierarchy.

The Table of Contents is configured to only list chapters and sections. We do not recommend including (sub-)subsections as this results in a very long Table of Contents, which may become difficult to read. If you do want to change the depth of the Table of Contents, you can change the value letocsettocdepth in setup.tex.

Use title case in chapter, section, (sub)subsection, and paragraph environments. Consider, for instance, https://capitalizemytitle.com for common title case capitalization rules.

Within a section, you should walk the reader through your text:

In the following, we describe the three primitives of concept $\mathrm{X}$. First, $\mathrm{X}$ uses the $\mathrm{Y}$ algorithm....

The second concept is $\mathrm{Z}$...

\subsection{Typography}

Use emphasis sparingly. This advice is especially true for bold print (\textbf\{bold print\}). ${ }^{15}$ Resist the urge to use it in the main text; use italics (\emph\{italics\}) instead.

Note, however, that too frequent use of italics can also be annoying. We recommend using it to emphasize ordinary words that are used as special terms - and only at its first occurrence. Also, you should emphasize words that you would stress when reading aloud, indicating that they are especially relevant for the meaning. Do not mix italics and quotes for emphasis, because this confuses the reader. ${ }^{16}$

Generally, we recommend avoiding scare quotes. ${ }^{17}$ Some authors use scare quotes to signal that they are using a word in a non-standard, ironic, or otherwise special sense (cf. Chicago Manual of Style). Scare quotes convey an informal tone. Moreover, they cause ambiguity, as the reader cannot be sure about the author's intention.

You should split your content into proper paragraphs by adding empty lines between adjacent blocks of text in the source file. Using a mixture of paragraphs and line breaks, which you could create with $\backslash$ at the end of a line, is strongly discouraged because this practice creates a noisy layout.

Use thin spaces $(\backslash$,$) in the appropriate places. For instance, write i. \backslash$, e., to obtain "i. e.," (pronunciation: "that is"). The same applies to "e. g.," (pronunciation: "for example"). These two abbreviations are normally followed by a comma in American English.

Dashes can be used instead of colons or pairs of commas to mark off a nested clause. Use an en dash for that purpose - like in this example. If you cannot type an en dash on your keyboard, you can write two regular hyphens next to each other. LaTeX will substitute them with an en dash. 
While we have no strict preference, we recommend using en dashes instead of the longer em dashes. If you use em dashes (-), you should not enclose them in spaces-as shown in this example sentence.

Use en dashes for ranges, e. g., when you write something like 5-10 \% (note the thin space before the \% symbol).

Use a proper minus symbol (e.g., by using math mode like this -1.337). Also, use a sensible number of digits after the decimal point.

Have you noticed that the main text uses old-style figures for numbers (12345), while figures in Math Mode and tables use lining figures (12345)? This difference is intended behavior. Note, however, that it is discouraged to print numbers with differing figure styles next to each other (as we are doing in this section). You may, therefore, have to enforce the use of lining figures. ${ }^{18}$ Lining figures can be enforced by enclosing the respective part in \textlf $\{. .$.$\} or by using Math Mode.$

Use proper directional quotation marks like "these." Directional quotation marks are created ' 'like this' ', by using \enquote\{text\}, or by copy-and-pasting the respective Unicode characters. Be aware of the following convention, which differs from German texts: The closing quotation mark is placed after any subsequent punctuation, e. g., "like this," and "like that."

Further Reading If you understand German, consider TYPOKURZ (ht tps://zvisionwelt.wordpress.com/downloads/), a short introduction to typography. You can also browse Matthew Butterick's website on typography (https://practicaltypography.com). The authoritative book on the matter is "The Elements of Typographic Style" by Robert Bringhurst.

\subsection{Language and Style}

Language issues distract readers from the content and make it difficult to assess its merits. You should, therefore, pay close attention to language and style.

\subsubsection{Spelling, Hyphenation, and Grammar}

Ensure correct spelling throughout your text. Check your writing with a spell checker. There are special spellcheckers for LaTeX, but copy-andpasting the text into Word may also be an option.

Ensure proper hyphenation throughout your text. You will have to intervene, for instance, when words extend into the margin of the page (creating so-called overfull hboxes). You can override LaTeX's hyphenation rules by inserting $\backslash$ - into a word. This special character indicates a conditional hyphenation point.

Often, it makes sense to define custom hyphenation rules globally. To this end, define your custom hyphenation definitions with \hyphenation\{FORTRAN Hyphen-a-tion\} and insert them after the Jbegin\{document\} clause. The ex-
18: Information on changing this default can be found in the documentation of the Cochineal font, which we use in this template: https://ftp.a gdsn.de/pub/mirrors/latex/dante/fonts/cochi neal/doc/cochineal-doc.pdf.
Oh no, look, an overfull hbox! 
19: http://hemingwayapp.com, https://gramm arly.com, https://languagetool.org, and the command-line tool (https://github.com/devd/A cademic-Writing-Check).
20: George D. Gopen and Judith A. Swan. The Science of Scientific Writing. Appeared in American Scientist 78(6):550-558 (Nov-Dec 1990), available online at https://cseweb.ucsd.edu/ sw anson/papers/science-of-writing.pdf and https:// www.americanscientist.org/blog/t he-long-view/the-science-of-scientific-writing.

21: Homepage of the lessons: https://cgi.duke. edu/web/sciwriting/, PDF slides: https://cgi.d uke.edu/web/sciwriting/resources/201108_Du keScientificWritingWorkshop.pdf (these URLs could not be archived).

22: Copyright (๑) 1990 by Sigma Xi, The Scientific Research Society. Reprinted with the permission of American Scientist. ample definition prevents any hyphenation of the word FORTRAN and defines three hyphenation points for the word hyphenation.

Also, check your text for common grammar issues (cf. Sect. 2.7.2).

\subsubsection{Style}

This section contains selected pieces of advice on particular aspects and typical errors. For a more comprehensive treatment, we refer the reader to the paragraph Common Bugs in Writing at the end of this section.

The main goal of a thesis is to convey information without ambiguity. Write concisely and use a simple language. Avoid complex sentence structures, unnecessary words, and unnecessarily complicated words. A thesis is not the place to show off your mastery of grammar and vocabulary. There are various (mostly web-based and commercial) tools ${ }^{19}$ that can help you identify common issues in your text.

\subsubsection{The Science of Scientific Writing}

Even if a text uses a simple language, it may be difficult to read, because it does not coherently convey the writer's train of thoughts. In short, your goal is to connect every sentence explicitly to its predecessor - which is easier said than done.

An excellent resource to develop this skill is the article The Science of Scientific Writing by George D. Gopen and Judith A. Swan. ${ }^{20}$ The advice from this article is also part of the three lessons on scientific writing offered by Duke University. ${ }^{21}$

The remainder of this section contains selected excerpts from The Science of Scientific Writing. ${ }^{22}$ Consider reading the original article for more extended treatment, including worked examples.

Subject-Verb Separation (Excerpts) Readers expect a grammatical subject to be followed immediately by the verb. Anything of length that intervenes between subject and verb is read as an interruption, and therefore as something of lesser importance. The reader's expectation stems from a pressing need for syntactic resolution, fulfilled only by the arrival of the verb. Without the verb, we do not know what the subject is doing, or what the sentence is all about.

As a result, the reader focuses attention on the arrival of the verb and resists recognizing anything in the interrupting material as being of primary importance. The longer the interruption lasts, the more likely it becomes that the "interruptive" material actually contains important information; but its structural location will continue to brand it as merely interruptive. Unfortunately, the reader will not discover its true value until too late - until the sentence has ended without having produced anything of much value outside of the subject-verb interruption.

The Stress Position (Excerpts) It is a linguistic commonplace that readers naturally emphasize the material that arrives at the end of a 
sentence. We refer to that location as a "stress position." Beginning with the exciting material and ending with a lack of luster often leaves us disappointed and destroys our sense of momentum.

The stress position can change in size from sentence to sentence. Sometimes it consists of a single word; sometimes it extends to several lines. The definitive factor is this: The stress position coincides with the moment of syntactic closure. A reader has reached the beginning of the stress position when she knows there is nothing left in the clause or sentence but the material presently being read.

To summarize the principles connected with the stress position, we have the proverbial wisdom, "Save the best for last."

The Topic Position (Excerpts) To summarize the principles connected with the other end of the sentence, which we will call the topic position, we have its proverbial contradiction, "First things first." In the stress position the reader needs and expects closure and fulfillment; in the topic position the reader needs and expects perspective and context.

The information that begins a sentence establishes for the reader a perspective for viewing the sentence as a unit: Readers expect a unit of discourse to be a story about whoever shows up first. "Bees disperse pollen" and "Pollen is dispersed by bees" are two different but equally respectable sentences about the same facts. The first tells us something about bees; the second tells us something about pollen. In fact, "Pollen is dispersed by bees" is the superior sentence if it appears in a paragraph that intends to tell us a continuing story about pollen. Pollen's story at that moment is a passive one.

Readers also expect the material occupying the topic position to provide them with linkage (looking backward) and context (looking forward).

\subsubsection{Selected Syntactical Conventions}

Avoid using informal contractions such as can't, don't, and it's. Replace them with cannot, do not, and it is.

We recommend using the serial comma in all lists to avoid ambiguity. The serial comma is also known as the Oxford comma: Insert it right before the word and that leads the last item of a list. The following example ${ }^{23}$ illustrates the benefit of using a serial comma:

Three important techniques in the design of algorithms are bisection, divide and conquer, and recursion.

Use bullet lists correctly. "Lists are common in all forms of writing. The list items can be included within the text or put on separate lines. Separate lines are used in order to draw attention to the items, to ease reading when the items are long or numerous, or to facilitate cross-reference to specific items." ${ }^{24}$ The environments itemize and enumerate produce lists on separate lines. It is considered good style to use punctuation in such a way that the lists form full sentences if their items were not split into separate lines. One way to achieve this goal is to put full sentences into the
23: Source: https://nhigham.com/2016/02/16/t he-serial-or-oxford-comma/

24: Source of cited text: https://nhigham.com /2015/12/17/punctuating-lists/. 
25: Available at http://www.cs.joensuu.fi/pages /whamalai/sciwri/sciwri.pdf.

26: Available at https://developers.google.com /tech-writing. list. If list items, however, are sentence fragments, additional punctuation is necessary (example taken from the source mentioned in the sidenote):

We used three different algorithms in the experiments. The table reports the performance of

- Algorithm 3.1 (based on a Taylor series),

- Algorithm 3.2 (with parameter $k=1$ ), and

- Algorithm 3.3 (with tolerance $10^{-8}$ ).

Common Bugs in Writing Read the comprehensive list of common bugs in writing by Henning Schulzrinne, which is available at http://w ww.cs.columbia.edu/ hgs/etc/writing-bugs.html.

\subsection{Concluding Remarks}

Scientific work and writing are skills that you can practice. Seminar papers serve that purpose during the studies. The final thesis shows which methodical and technical skills you have acquired. In addition to proper time management, discipline, and willingness to research literature, communication with one's supervisor is the key to success.

Further Reading This guide is not meant to cover all topics of scientific writing in detail. Consider the very comprehensive document Scientific Writing for Computer Science Students by Wilhelmiina Hämäläinen. ${ }^{25}$ Hämäläinen has collected a substantial amount of advice, focusing on English grammar and the peculiarities of computer science.

If you are pressed for time, consider the pre-class parts of "Technical Writing Courses for Engineers" by Google. ${ }^{26}$ The pre-class parts cover essentials and are split into short sections taking less than 15 minutes each. There is also an abundant number of books on scientific writing. We recommend (available in print and as e-book in many libraries):

- M. Alley. The Craft of Scientific Writing; and

- W. Strunk and E.B. White. The Elements of Style.

Finally, if you seek inspiration, we recommend to read these reports by well-known scientists:

- Randy Pausch: Time Management (http://www.youtube.com/watch ?v=oTugjssqOT0),

- Richard Hamming: You and Your Research (http://www.cs.virginia.e $\mathrm{du} / \sim \operatorname{robins} /$ YouAndYourResearch.html), and

- Nick Feamster: Writing Tips for Academics (http://greatresearch.org /2013/10/11/storytelling-101-writing-tips-for-academics/).

The rest of this guide provides some general advice (Appendix A), advice on presenting figures and tables (Appendix B), and less-often needed technical details about the template (Appendix C). 


\section{A Further Recommendations}

This appendix contains further recommendations, which our students found useful in the past.

\section{A.1 Introduction, Related Work, and Conclusion}

\section{A.1.1 Introduction Chapter}

Don't be boring! Pull in the reader with a peculiar observation or a surprising result of your thesis.

Approaches to structure your introduction:

- Start with the general, close with the specific.

- Start with what is already well-known, move on to what has only recently become known.

- State the objective of the thesis, then describe your approach.

End the introduction with a paragraph Outline of the Thesis that describes its structure in prose.

\section{A.1.2 Related Work Chapter}

Think about a story and which message you want to convey. Some common themes are:

- "It used to be a difficult problem, but we know how to solve it."

- "While a lot of proposals exist, none has gained traction in reality. X found that one of the primary reasons for that situation is ..."

- "The field is a cat-and-mouse game between refined attacks and defenses. A common theme is ... and overlooked areas are ..."

- "While most of the current literature focuses on X, it would make sense to apply techniques from field $\mathrm{Y}$ to the problem. One could then refine the problem as...” 


\section{A.1.3 Conclusion Chapter}

A basic recipe for a conclusion chapter:

- a summary,

- a critical assessment of what you have achieved,

- pointers to related other topics,

- a statement of the impact of the results, and

- pointers to future work.

\section{A.2 Thesis Length}

Typical theses range from 25 to 150 pages. There have been excellent theses at both ends of this spectrum. Do not worry too much about the page count. Write everything that is necessary to assess and reproduce your work, but no more. Less is more!

Check the individual parts for an appropriate length: Do not write five pages in the introduction if the main part has only 15 pages.

\section{A.3 Practices to Avoid}

- Colloquial style: "Computation power has skyrocketed in the last few years."

- Widespread knowledge: "The Internet is becoming more and more important."

- Superlatives: "One of the most beautiful concepts," "great possibilities."

- Be careful with strong claims: "the only possibility," "the best solution," "impossible." 


\section{B $\quad$ Designing Figures and Tables}

In Section 2.3, we have shown how you can integrate tables, figures, and listings into your thesis. In this appendix, we give recommendations on a higher level. In the following sections, we focus on designing compelling figures and tables, i. e., how you can present material effectively. For figures, we will cover both graphs as well as diagrams.

We have compiled most of the content in this appendix from the following two books (permission to reproduce the respective figures has been obtained from Elsevier):

- the book Designing Science Presentations: A Visual Guide to Figures, Papers, Slides, Posters, and More by Matt Carter, ๑ 2012 [Car12] and

- the book Information Visualization: Perception for Design by Colin Ware, (c) 2012 [War12].

Another useful resource is the book Universal Principles of Design: 125 Ways to Enhance Usability, Influence Perception, Increase Appeal, Make Better Design Decisions, and Teach Through Design [Lid+1o].

\section{B.1 Fundamental Concepts}

We start by revisiting the fundamentals of visual perception as well as information organization. The following section will apply the concepts shown in this section.

When you design figures, you should be aware of the Gestalt laws and the HSB color model, which will be described in the following two sections. After that, we will revisit the basics of information organization, which apply to figures and tables alike.

\section{B.1.1 Gestalt Laws}

The Gestalt laws have been known since the early 190os. They describe how we perceive patterns. In the following, we will discuss the following Gestalt laws: proximity, similarity, connectedness, continuity, symmetry, closure, and relative size.
While this guide is published under a Creative Commons license (cf. Sect. C.3), this license does not apply to the reproduced figures. Elsevier and/or the authors hold the copyright for the reproduced figures.

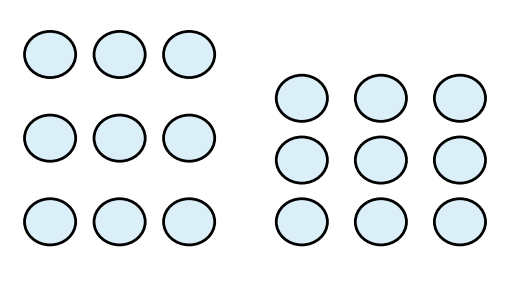

Figure B.1: Spacing makes us perceive rows or columns (reproduced from [War12] with permission). 
Figure B.5: We can spot differences in these age distribution plots faster when data is plotted symmetrically (Data source: https://destatis.de, own illustration).
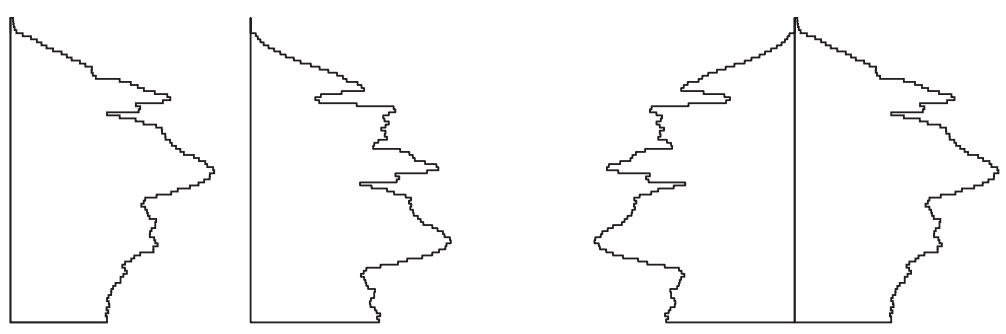

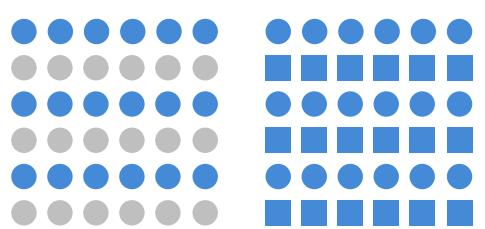

Figure B.2: We perceive similar elements as a group (reproduced from [War12] with permission).

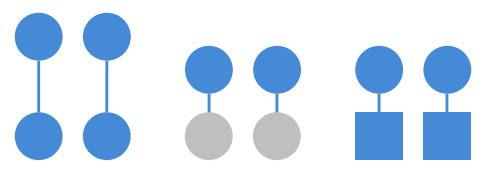

Figure B.3: Connections are more powerful than similarity (reproduced from [War12] with permission).
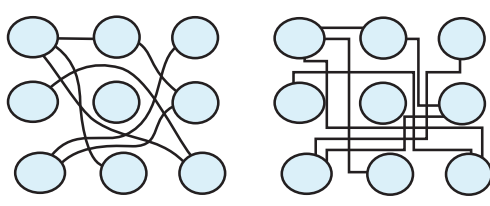

Figure B.4: Continuity makes the left-hand diagram easier to read (reproduced from [War12] with permission).
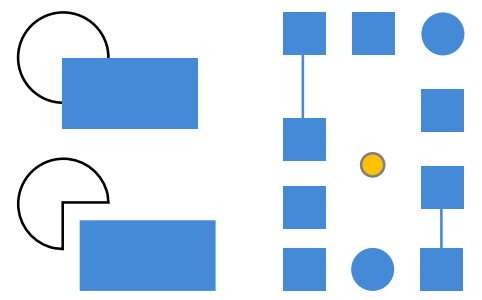

Figure B.6: Left: closure makes us perceive a full circle (reproduced from [War12] with permission); right: the orange dot is perceived to sit inside of a rectangle.
The first law, proximity, refers to the spatial relationships between groups of objects. Objects that are closer together form a group. We are very sensitive to spatial relationships. Even small changes in spacing can change our interpretation of a scene (cf. Fig. B.1). Therefore, you should "place symbols and glyphs representing related information close together." [War12]. The proximity law is the reason why adding additional vertical space between (groups of) rows helps us with reading a table.

Similarity is the second Gestalt law. Visual similarities such as color or shape allow us to identify groups of objects with ease (cf. Fig. B.2). Large tables, for instance, can benefit from alternated shading of rows. A downside of shading is the additional visual clutter. We prefer to add extra space every three to five rows to keep tables readable. The similarity law is also essential in diagrams that use different shapes or styles. Visual differences help the reader group similar elements.

Connectedness is a powerful principle that is stronger than proximity, shape, and style (cf. Fig. B.3). Consider connecting related objects with lines.

The next principle, continuity, "states that we are more likely to construct visual entities out of visual elements that are smooth and continuous, rather than ones that contain abrupt changes in direction" [War12]. It is, therefore, not surprising that node-link diagrams with smooth lines are easier to read than those with straight lines (cf. Fig. B.4).

Another principle is symmetry. Symmetrical figures are visually pleasing, and we are good at detecting asymmetry. Symmetry is a form of high-level similarity. If you organize groups of elements in a diagram symmetrically, readers will assume that this means that they are similar. If you design diagrams asymmetrically, the asymmetric parts get emphasized. Our ability to check for symmetry can also be useful during visual data analysis (cf. Fig. B.5).

We are also on the lookout for closure and common ground. Closed contours are perceived as objects. Our search for closure is so strong that our brain interpolates missing parts of contours to form whole objects. As a result, we see a rectangle and a complete circle on the left-hand side of Fig. B.6 instead of a circle with a missing segment. Moreover, contours create a notion of common ground with an "inside" and an "outside".

Common grounds are perceived for complete contours as well as for contours that we perceive due to closure. Thus, on the right-hand side of 


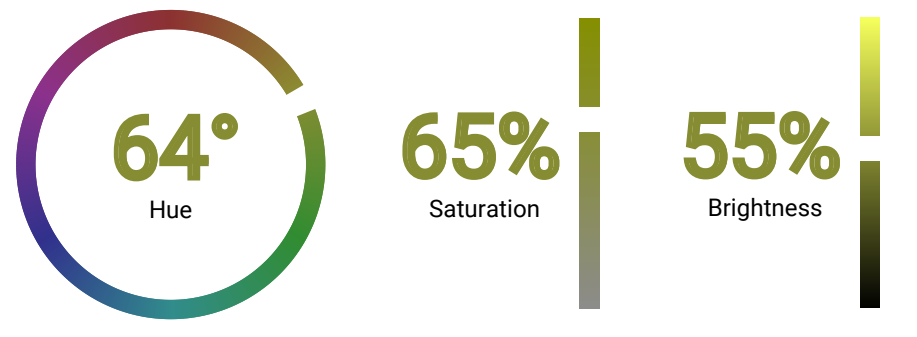

Fig. B.6, we perceive the orange dot to be inside an imaginary rectangle built by shapes. Ware recommends: "Consider putting related information inside a closed contour. A line is adequate for regions having a simple shape. Color or texture can be used to define regions that have more complex shapes" [War12].

The final Gestalt law to discuss is figure and ground. Figures are perceived as objects that are positioned in the foreground. The ground lies behind the figures. We perceive an object as a figure when it consists of a closed contour that forms a common ground and is considerably smaller compared to its surroundings.

This principle is important when drawing diagrams that use contours to demarcate the boundaries of various systems. When multiple systems are nested, and the size differences are too small, it can become difficult to perceive the difference between figure and ground. Consider the diagram shown in Fig. B.7). On the left-hand side of this diagram, too many rectangular shapes have been nested. Due to equal amounts of whitespace around every shape, multiple interpretations are possible. The right-hand side of the diagram is slightly better because the circles are considerably smaller than the surrounding rectangle. Moreover, together with the arrows the circles are perceived as a (symmetric) shape, which is easily perceived as a figure sitting on the rectangle in the background.

\section{B.1.2 Color}

You are probably used to defining colors in terms of red, green, and blue (RGB) or cyan, magenta, yellow, and black (CMYK). A third way, which is more useful, is the HSB model. It defines color in terms of hue, saturation, and brightness. ${ }^{1}$ Consider the color picker shown in Fig. B. 8 for an example.

The first value, hue, defines the color according to its position (given in degrees ranging from o to 360) on the color wheel. For instance, the value of 60 corresponds to the hue yellow. Saturation (o to 100) corresponds to the richness of the color, where o means that there is no trace of the hue, i. e., a gray color between white and black. The value 100 means that the hue is fully present, i. e., the color is as colorful as possible. The final value is brightness (o to 100). A value of o corresponds to a solid black, a value of 100 corresponds to the brightest version of the hue at the given saturation.

Different colors are often used to ease visual perception (cf. Fig. B.9). For
Figure B.8: A HSB color picker (https:// codep en.io/HunorMarton/full/eWvewo)

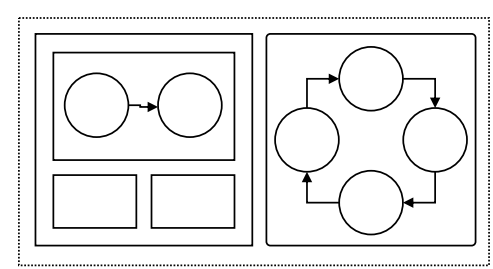

Figure B.7: Figure and ground are difficult to pick apart in this diagram; also note how PowerPoint fails to draw straight connectors (own illustration).

1: Read the primer at https://learnui.design/blo g/the-hsb-color-system-practicioners-primer.h tml for more details.

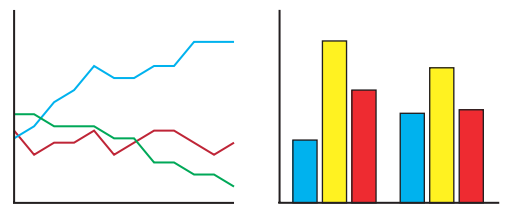

Figure B.9: Using colors to ease visual perception (reproduced from [Car12] with permission). 


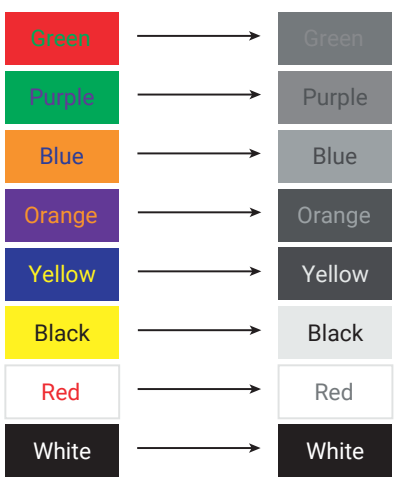

Figure B.10: Colors in monochrome (reproduced from [Car12] with permission)

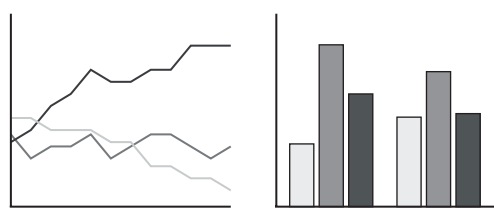

Figure B.11: Shades of gray can be very effective (reproduced from [Car12] with permission).

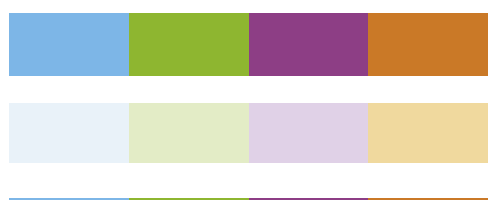

Figure B.12: Use less saturation for large shapes, and more for thin lines (reproduced from [War12] with permission) instance, it can be used for emphasis, to group subsets of elements, or to make it easier to distinguish different elements that have a similar shape.

Be aware of the monochrome representation of colors, which makes it impossible to distinguish a subset of colors. Red and green are particularly difficult to differentiate in monochrome print, especially if they have the same saturation and brightness (cf. Fig. B.10).

The risk of confusion in monochrome prints is not the only reason why changing the hue is problematic. Different hues are difficult to make out when the area is small, as in the line plot in Fig. B.9. Moreover, different colors (hues) have different connotations (such as green means good, red means danger). Finally, different colors have a different visual weight that may create unwanted emphasis (blue is heavier than orange).

Therefore, it is often better to stick with one hue and use different levels of brightness or saturation to ease visual perception. Figure B.11 uses three shades of gray and is easier to read than the colorful version in Fig. B.9.

When you use more than four different grayscale colors, however, the differences become too small to perceive with ease. Too many grayscale colors are especially problematic when color is used on its own, such as in line plots. It is less problematic in bar plots, because the bars can be sorted with decreasing brightness levels to create (good) redundancy. If brightness is used to encode values of data, darker colors should be used for higher values.

Similar principles apply for saturation: "If using color saturation to encode numerical quantity, use greater saturation to represent greater numerical quantities. Avoid using a saturation sequence to encode more than three values" [War12]. Consider varying both brightness and saturation at the same time to create shades that are easier to distinguish from another.

The effects of different levels of saturation vary depending on the size of the colored area (cf. Fig. B.12). If you consider varying saturation, stick to the following guidelines: "Use more saturated colors when color coding small symbols, thin lines, or other small areas. Use less saturated colors for coding large areas" [War12].

\section{B.2 Organizing Information}

In this section, we will review selected design concepts to organize information in a meaningful way. The book by Lidwell et al. contains more information on the ideas [Lid+10].

\section{B.2.1 Advance Organizer}

An often-cited principle for giving a speech is summarized as follows: first, you announce what you are going to talk about; second, you talk about that; third, you review what you talked about. A design concept 
that implements this advice is an advance organizer. Advance organizers are short chunks of information that are presented before new material. This way, readers will find it easier to learn new concepts.

Note that an advance organizer is not just a summary or an abstract. Its purpose is to relate new pieces of information to previously covered material and explain how this fits into the big picture. There are two kinds of advance organizers: expository and comparative.

Expository advance organizers, as Lidwell et al. [Lid+10] explain, "are useful when audiences have little or no knowledge similar to the information being taught." Expository advance organizers are effective when they provide a concise overview of the new material and how it fits together and into the big picture.

On the other hand, comparative advance organizers "are useful when audiences have existing knowledge similar to the information being presented. [They] compare and contrast features and operations between the familiar and the new" $[\mathrm{Lid}+10]$.

You can create advance organizers in the form of diagrams or as plain text. As a first step, you can add a signpost paragraph at the beginning of chapters and sections.

\section{B.2.2 Hierarchy}

Complicated relationships are often explained using a hierarchical approach. There are three basic ways to represent hierarchy: trees, nests, and stairs (cf. Fig. B.13).

Trees are useful to visualize structures that consist of parent-child relationships such as A dominates B and $\mathrm{C}, \mathrm{B}$ dominates of $\mathrm{D}$ and $\mathrm{E}$, and so on. Usually, child elements are located below or to the right of their parents. As Lidwell et al. [Lid+10] observe, "tree structures grow large quickly, and become tangled when multiple parents share common child elements. Tree structures are commonly used to represent overviews or high-level maps of system organization."

Nest visualizations are especially useful in visualizing parent elements that contain child elements. Nest visualizations can be challenging to read when relationships are complicated.

Finally, stair structures stack child elements below and to the right of parent elements. Stair visualizations are common in outlines. They can be used to capture arbitrarily complex hierarchies, especially when used interactively and individual nodes can be collapsed. Stair visualization also has a disadvantage: it may falsely imply a sequential relationship between the stacked child elements.

\section{B.2.3 Five Ways of Organization}

The so-called Five Hat Racks design principle suggests five common ways to organize information: by category, time, location, alphabet, and continuum $[\mathrm{Lid}+1 \mathrm{O}]$.
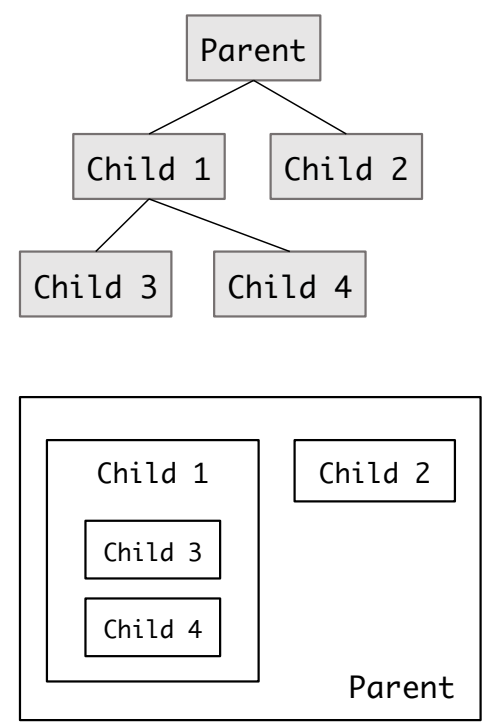

\section{Parent}

Child 1

Child 3

Child 4

Child 2

Figure B.13: You can visualize hierarchies with trees, nests, and stairs (own illustration). 


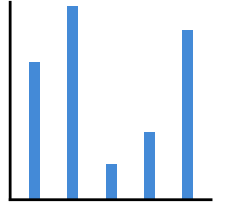

A $B$ C $\quad$ D $\quad E$

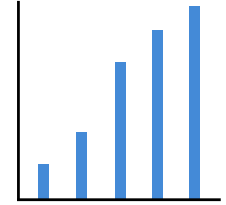

C D A E B
Figure B.14: Bar plots benefit from ordering the bars by length, an application of organization by continuum (own illustration).
1. Organization by category relies on similarity or relatedness of elements. Sometimes, categories can be organized hierarchically fashion.Note, Category is a nominal attribute, i. e., there is no natural order. If categories have to be ordered, you can rely on alphabetical order or use an apparent order such as "start with all the normal categories, followed by the special ones."

2. Organization by time is based on some chronological sequence. Ordering by time feels very natural. Note, however, that a chronological organization is not always useful. A literature review that merely presents one paper after another chronologically will not generate much insight.

3. Organization by location relies on geographical or spatial properties of the elements. Location can also be purely a logical concept, for instance, components that make up a system (which is also a hierarchy). It is common to describe systems by explaining one component (location) after another.

4. Alphabetical organization is another common technique. As Lidwell et al. [Lid+1o] remark, "organize information alphabetically, when information is referential, when efficient nonlinear access to specific items is required, or when no other organizing strategy is appropriate.”

5. Organization by continuum relies on a numeric property of the elements (e. g., ordering from highest to lowest or best to worst). Lidwell et al. [Lid+10] recommend to "organize information by continuum when comparing things across a common measure." Organization by continuum can improve the readability of bar plots (cf. Fig. B.14)

\section{B.3 Diagrams}

You can use diagrams to describe concepts and their relationship, the structure of systems, interactions, and (experimental) procedures.

\section{B.3.1 Before You Start}

Your first task is to decide whether a visualization makes sense at all. Sometimes it makes sense to choose a text-only representation such as pseudocode instead of a diagram. Ware [War12] shares the example of a flow chart, which is supposed to make it easier to understand the program flow (cf. Fig. B.15). He argues that pseudo code is superior. After all, the flow chart takes more effort to parse than the natural language used in the pseudo code (and, as Edward Tufte would argue, the flow chart contains more visual clutter than the pseudo code).

On the other hand, Ware argues, there are concepts that we can grasp much faster if we see a visual representation. Consider the following statements about a management hierarchy [War12]:

- Jane is Jim's boss.

- Jim is Joe's boss. 


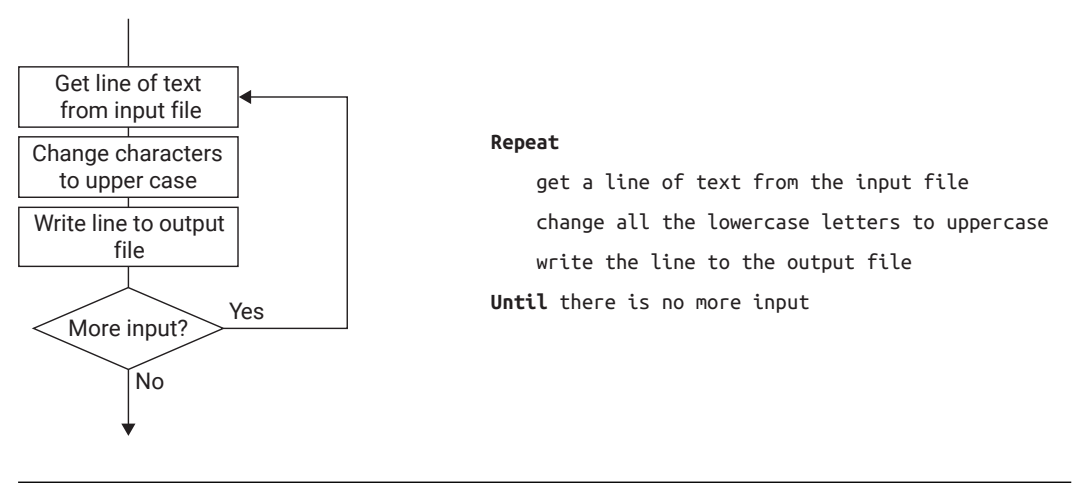

- Anne works for Jane.

- Mark works for Jim.

- Anne is Mary's boss.

- Anne is Mike's boss

It is challenging to keep track of all relationships in this presentation. You might even feel the urge to draw a tree. And, indeed, a graphical representation is much more accessible (cf. Fig. B.16). Ware concludes: "Diagrams should be used to express structural relationships among program elements, whereas words should be used to express detailed procedural logic" [War12].

Now, if you decide that you do want to create a diagram, you should ask yourself the following questions [Car12]:

- What is absolutely necessary to show?

- What is not necessary to show?

- What is most important and should be emphasized?

- What is not important and should be secondary to the main message?

- What are the relationships between individual elements?

- Does the diagram require a precise depiction of time?

- Does the diagram require a precise depiction of distance?

- What symbols should be consistent throughout the diagram?

In the following sections, we will explain the most important aspects to create useful diagrams.

\section{B.3.2 Elements and Relationships}

According to the Gestalt laws, you should "use small, closed shapes to represent data entities, and use the color, shape, and size of those shapes to represent attributes of those entities" [War12]. Figure B.17 shows the effect of different properties of shapes.

Many diagrams are supposed to visualize relationships of elements. Ware
Figure B.15: In comparison to pseudo code a flow chart is a poor representation of program flow (reproduced from [War12] with permission).

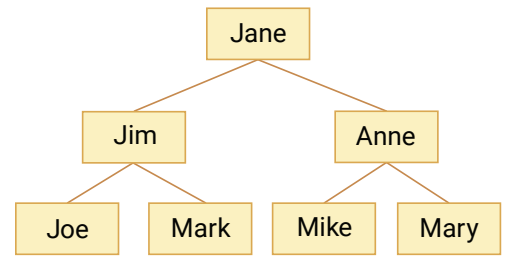

Figure B.16: A tree helps us grasp static relationships (reproduced from [War12] with permission). 
Figure B.17: Semantics of four properties of shapes (reproduced from [War12] with permission)
1. Closed contour: objects

2. Compact shapes: entity types
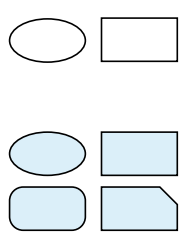

1. Partitioned region: entity partitions

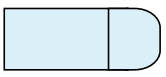

2. Attached shapes: part-of relationship

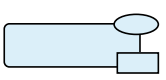

3. Enclosed shapes: contained elements

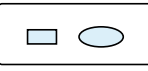

4. Sequence of shapes: sequence of entities

Figure B.18: Semantics of ten types of visual relationships (reproduced from [War12] with permission).
5. Linking line: generic relationship
3. Color of shape: entity types

4. Size of shape: entity value

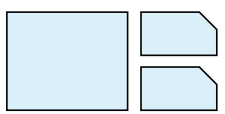

6. Asymmetric line: asym. relationship

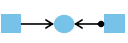

7. Line style: type of relationship

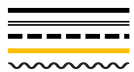

8. Line weight: strength of relationsh.

9. Shapes with receptables: fit between components

10. Proximity groupings: groups of components

recommends to use "connecting lines, enclosure, grouping, and attachment to represent relationships between entities. The shape, color, and thickness of lines and enclosures can represent the types of relationships" [War12]. Figure B.18 visualizes ten alternatives and how we perceive them.

Of note are the tapered lines in Number 6 of Fig. B.18. As explained by Ware, these are easier to recognize than arrows [War12], especially in busy diagrams. If you only use straight lines, you can use skinny triangles to create tapered lines. The broad end is located at the source of the line.

For more complex lines, you need a vector drawing tool like Inkscape, Adobe Illustrator, and Affinity Designer. Such tools also allow you to draw the wiggly line shown in Number 7 of Fig. B.18. Most drawing tools enable you to create shapes with receptacles (cf. Number 9 of Fig. B.18) by creating unions and differences of shapes.

\section{B.3.3 Emphasizing Elements}

Useful diagrams are self-explanatory and guide the reader's attention. Humans continuously search for patterns and deviations. Consistent use of shapes and colors indicates that the presented elements are similar (cf. Fig. B.19). Deviations from the norm indicate differences that need attention. Be aware of that, and do not create emphasis unintentionally.

Do not choose the size of elements arbitrarily. Differences translate into dominance relationships. Larger elements usually appear to control the smaller ones (cf. Fig. B.20) 

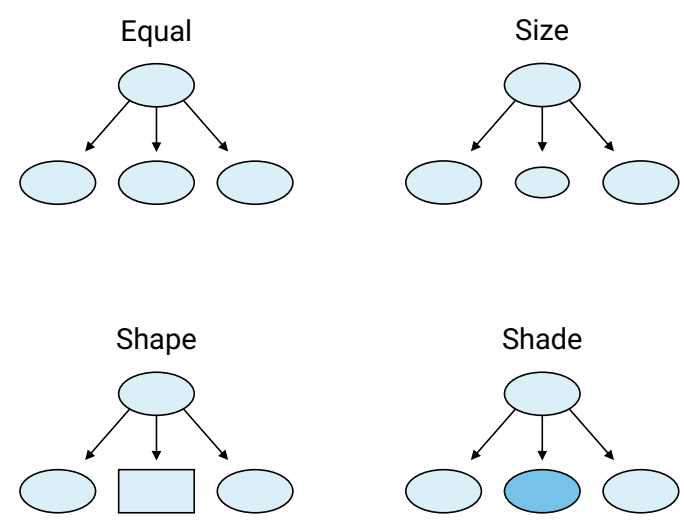

Parent dominant

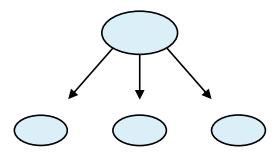

Child dominant

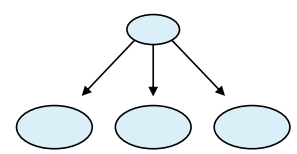

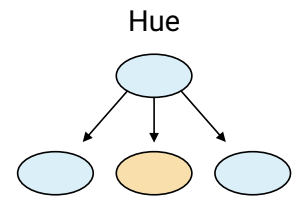

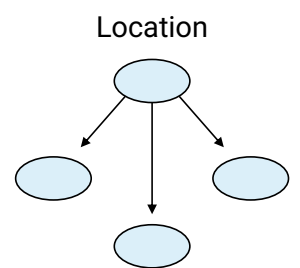

Child dominant with emphasis

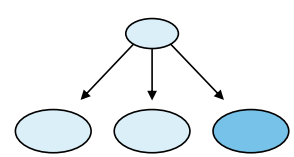

Figure B.19: Deviations from the norm create emphasis (reproduced from [Car12] with permission).

\section{B.3.4 Layout}

In the absence of strong emphasis, readers process diagrams similar to text (cf. Fig. B.21). In western cultures, readers will start in the top left corner and proceed horizontally in a zig-zag pattern. The general flow of information should be consistent with this expectation.

Ensure that all elements of a diagram are correctly aligned. Alignment helps readers to grasp the overall structure. Proper alignment can also save you from creating additional outlines to depict (systems) boundaries - proximity and neat alignment can create strong cohesion by themselves (closure).

Make conscious decisions about distances and dimensions (proximity, repetition). You can use a grid to enforce consistent distances. Note, however, that snapping every element to the grid lines, may still cause misalignment. ${ }^{2}$ Make use of the horizontal and vertical alignment tools that space out elements equally. An advanced technique is to create a dummy box shape to measure and compare dimensions yourself.

\section{B.3.5 Labels}

Many diagrams consist of shapes and lines, annotated with text labels.

A common technique is to use bold print to express some property of an element. For instance, the labels of shapes corresponding to systems are printed in bold to differentiate them from the shapes that correspond to exchanged messages. In general, we recommend avoiding this practice. Reserve bold print to emphasize one particular element in a diagram. Use another visual style to show differences, such as shading, colors, or

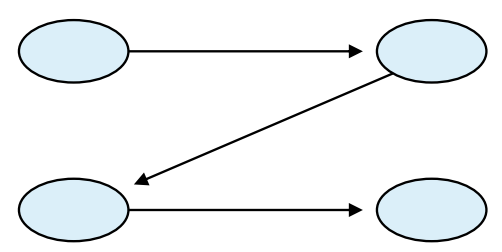

Figure B.21: Respect the expected flow of information in western cultures (reproduced from [Car12] with permission).

2: Consider a shape that is five grid lines high. Then, a horizontal line that leaves the box cannot be aligned in the middle.
Figure B.20: Relative differences in size indicate dominance relationships (reproduced from [Car12] with permission). 
Figure B.22: Moving the labels inside objects reduces clutter. Note how the left-hand-side figure is centered in the left part of the figure to keep the figure balanced (reproduced from [Car12] with permission).
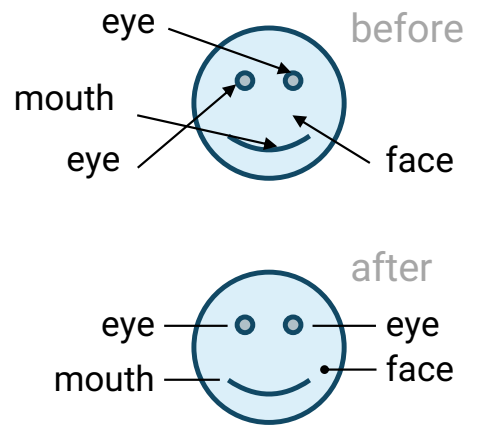

Figure B.23: Outside labels should not distract the reader (own illustration, inspired by [Car12]).

3: The advice in the following paragraphs has been compiled from https://www. slideshare. net/otikik/how-to-make-awesome-diagrams-foryour-slides/19-Size_doesnt_mean_long_name.
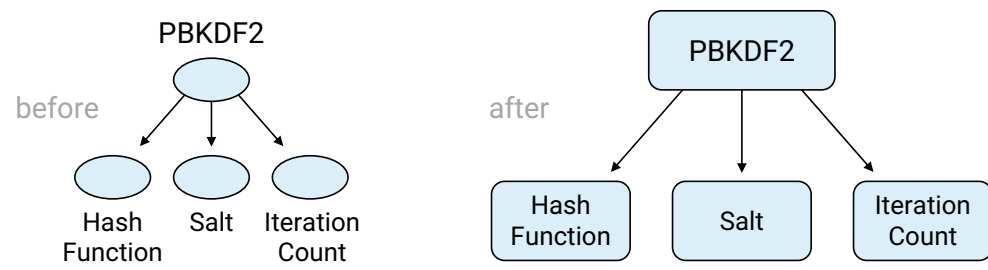

shape form. Consider giving an element no surrounding shape at all, for instance, if the notion of its boundary is not relevant or if the arrangement of sub-elements establishes a common ground due to the Gestalt law of closure.

In any case, the labels should be as close as possible to the shapes (Gestalt law proximity) and precisely aligned. Whenever possible, consider moving the labels into the shapes (Fig. B.22). Inside labels rely on the Gestalt law of common ground. They reduce visual clutter and make it easier to create a well-balanced diagram with no ragged edges (laws of symmetry and closure).

Figure B.23 illustrates critical principles when labels are outside of a shape. First of all, keep the lines as short as possible (proximity). Consider removing the arrowheads from the lines that point into an object to avoid confusion with other arrows in the diagram. Also, avoid crossing lines. Align labels on the left-hand side of an object flush right and vice versa (symmetry). Aim for consistency by making the lines parallel. Keep adequate amounts of surrounding whitespace.

Diagrams that visualize structures can work fine with few labels. In contrast, diagrams that visualize procedures or more complex relationships need more textual explanation. Consider adding longer descriptions right next to the corresponding locations (proximity) as shown in Fig. B.24.

\section{B.3.6 Variations}

Common drawing tools provide a large number of shapes. What is missing, however, is guidance on how to combine them reasonably. Figure B.25 displays eight combinations. ${ }^{3}$

The first variation in the left-hand column shows a style useful for depicting information exchange between systems, such as servers and clients. The second variation uses an arrow shape, which emphasizes the activity represented by the arrow. The third and fourth examples in the left-hand column depict two steps from a process without too much visual clutter. We prefer the latter one because the text wraps at sensible points.

The right-hand column shows variations without outlines. At first glance, this sounds like a good idea: it avoids visual clutter (the outlines). Note, however, that shapes without outlines need more saturated and darker color to differentiate them from the background. If the number of shapes in a diagram is large, this approach can make it more difficult to read the diagram because all shapes appear to be emphasized simultaneously. 


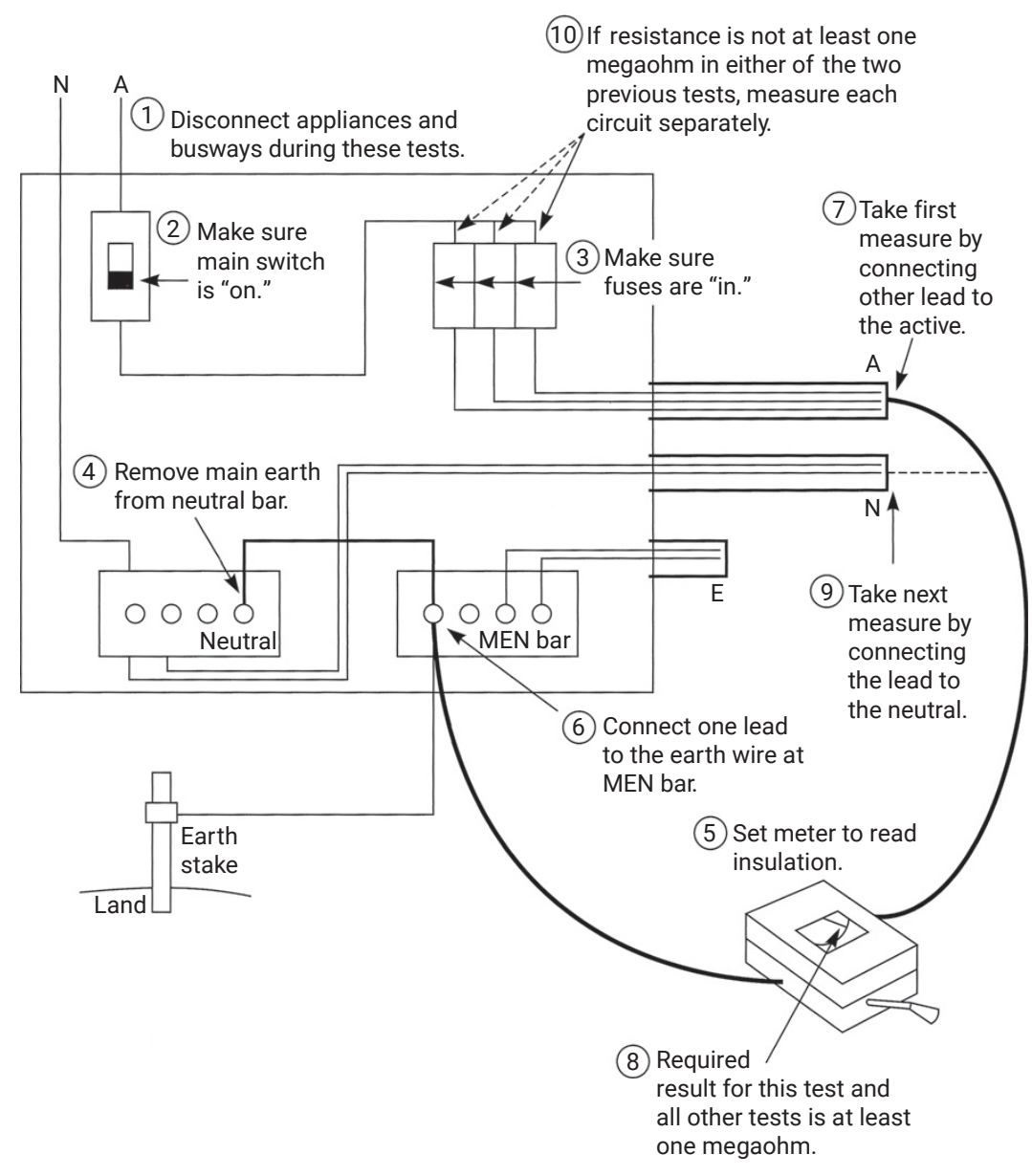

If you use dark shapes, you should avoid having white text on dark background for small font sizes. As shown in the second variation on the right-hand side of Fig. B.25, you will have to make changes to some shapes.

In any case, you should choose the sizes of shapes consciously. Represent objects with identical properties with shapes of the same size. If the labels do not fit into the shapes, you can either reduce the font size, put the labels next to the box, or increase the size of all shapes (by rearranging the diagram).

Finally, consider the option of using negative space, as shown in the bottom-right corner of Fig. B.25. Again, this can be a useful means to avoid visual clutter. However, if you increase the width of the surrounding white stroke (as was done for the example figure), the (vertical) padding in the shape will become too narrow. Moreover, you cannot easily align such shapes with other shapes with thinner strokes.

\section{B.4 Graphs}

In this section, we review common design principles for graphs (also called charts or plots). Besides our guidelines, we will present the examples shown by Carter [Car12].
Figure B.24: Complex diagrams benefit from integrated explanations. Source of figure as cited by [War12]: R Chandler and J. Sweller (1991). Cognitive load theory and the format of instruction. Cognition and Instruction, 8:4, 293-332, DOI: 10.1207/s1532690xci0804_2 www.tandfonline.com. This figure is not subject to the Creative Commons License under which this guide is published (cf. Sect. C.3); copyright is held by the publisher and/or the authors. 


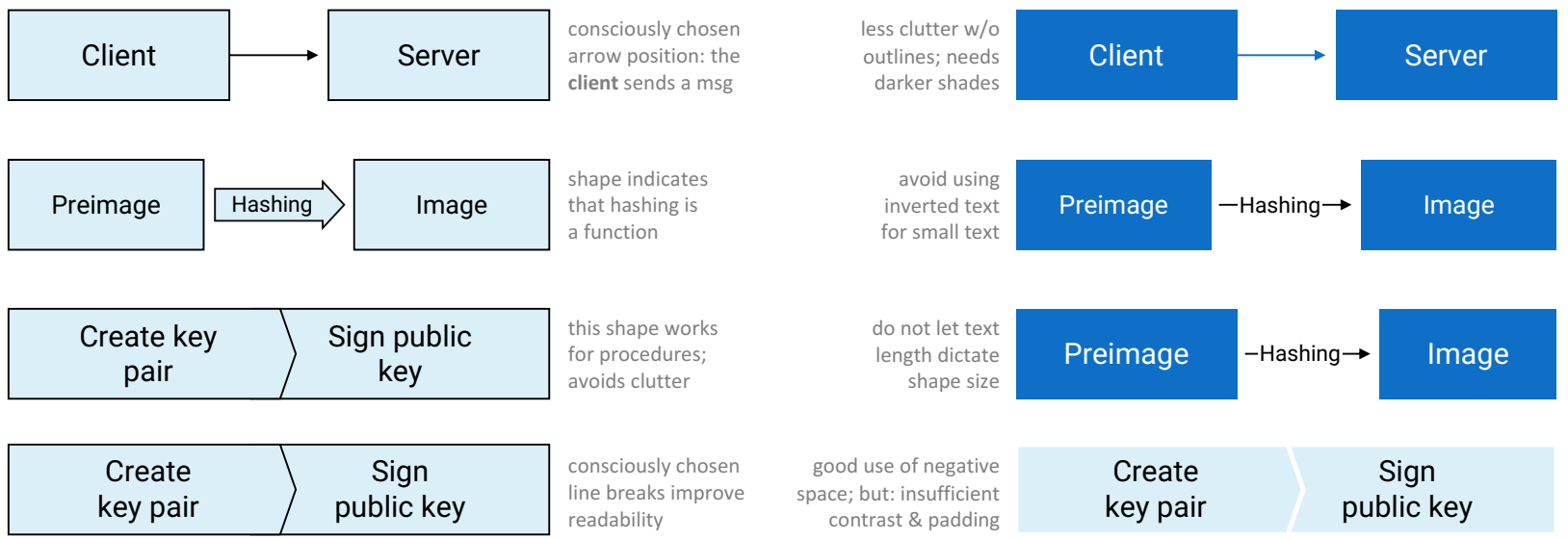

Figure B.25: Eight variations resulting from combining different outline, shade, and arrow styles.
4: For comprehensive overviews and guidance see https://datavizcatalogue.com, https: //depictdatastudio.com/charts/, https://w ww.data-to-viz.com/img/poster/poster_big.png and https://www.labnol.org/images/2008/datachart-type.png.
The first step in data visualization is to pick a reasonable chart type. Most visualization needs that arise in a thesis can be addressed with line charts, bar charts, and histograms, and scatter plots. There are many more chart types such as Chord Diagrams, Sankey Diagrams, and the Nightingale Rose Chart. ${ }^{4}$ These and other advanced chart types can be useful in particular situations. In general, however, we recommend sticking to more basic chart types. In particular, avoid pie charts unless you know what you are doing.

General advice for any graph is to have legible axis labels and, if more than one data series is visualized, a legend for the data series. Moreover, remember to use colors effectively (cf. Sect. B.1.2).

The following sections provide more information on two essential chart types, line charts and bar charts. The recommendations are very concrete. You may find that you cannot follow all of them using your plotting tool of choice. If this limitation bothers you, consider editing the plotting tool's output with a vector drawing program such as Inkscape or Adobe Illustrator.

\section{B.4.1 Line Charts}

Line charts show how one variable (the one on the y-axis) changes when another one varies within a given range. Line charts are particularly useful to visualize time series.

Often, there are many lines to be plotted. Resist the temptation to add more than three lines into one line chart if the lines overlap. Instead, create multiple charts that have one of the lines in common. This line serves as a baseline, easing comparison.

Figure B.26 summarizes Carter's advice on line charts.

\section{B.4.2 Bar Charts}

The top row of Fig. B.27 summarizes Carter's advice on bar charts. Bar charts are useful to compare the value of a single variable under different circumstances. The value of the variable corresponds to the length of a 

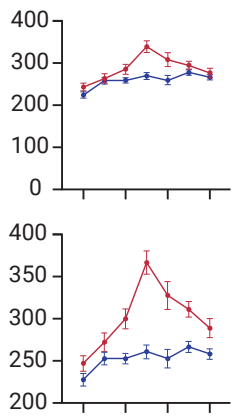

Range in height should be ca. three fourths the height of the y-axis (axis does not have to start at zero).
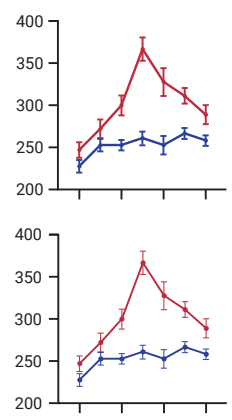

Line weights: about the same as the lines of the axes. Data points: at least twice as thick as the line itself. Error bars about three-fourths as thick.
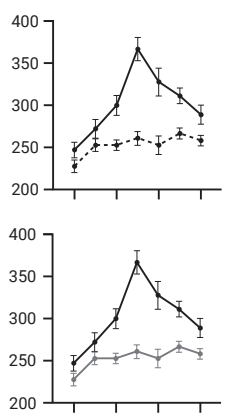

Dashed lines: hard to read; use colors! Alternative, not shown: different symbols. Filled • and outlined symbols easier to differentiate than different shapes.
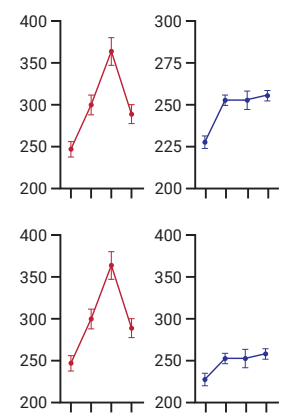

When presenting two charts next to each other that represent the same experiment, make sure your axes are similar in scale. Otherwise, you risk misrepresenting your data.

bar. In principle, a single bar chart can also show the values of different variables. Note, however, that such a chart is more challenging to read, especially when the variables need differently scaled Y-axes (one to the left and one to the right of the chart).

The bars can be drawn horizontally and vertically. ${ }^{5}$ It is common to use vertical bars for time series (time on the $\mathrm{x}$-axis). You can also use vertical bars if the values on the $\mathrm{x}$-axis are on an ordinal scale (i. e., they are arranged in their natural order). Vertical bars do not work well with long labels. Rotating labels makes them challenging to read. On the other hand, horizontal bar charts work well with longer labels (align the labels flush right next to the bars).

The advice in this section assumes vertical bars. In vertical bar charts, the $\mathrm{Y}$-axis corresponds to the value of the variable, and the $\mathrm{X}$-axis is typically used for a categorial variable, which represents the different circumstances.

An advanced version of a classical bar chart is a stacked bar chart, an excellent alternative to visualize proportions of a whole. In contrast, to pie charts, which can be deceiving, stacked bar charts are more accurate and can be easily compared.

Histograms A special kind of bar charts is a histogram. Carter gives a concise explanation of the purpose of a histogram: "A histogram shows the distribution of data and the relative frequency with which the data occur. It essentially offers the audience an estimate of the probability distribution of a dataset" [Car12]. The bottom row of Fig. B.27 summarizes Carter's advice on histograms.

\section{B.5 Tables}

Tables are useful to display multiple properties for several entities. This section summarizes Carter's guidelines for designing effective tables
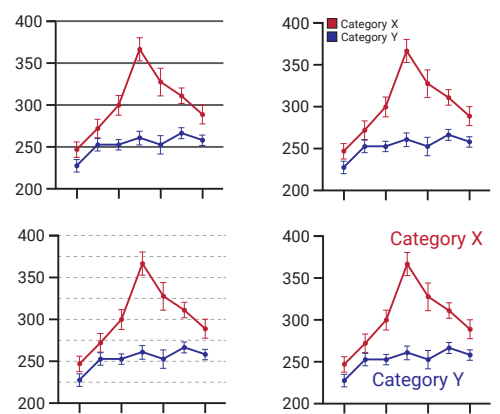

Don't overwhelm your chart with a distracting gridline.

If you feel a gridline

is helpful, place a

subtle gray grid in

the background.

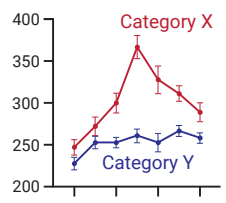

Try to place your data labels next to

the lines them-

selves, rather than

in a far-off spot.
Figure B.26: Advice for line charts (reproduced from [Car12] with permission).

5: See https://depictdatastudio.com/when-to-u se-horizontal-bar-charts-vs-vertical-column-char ts/ for more guidance. 

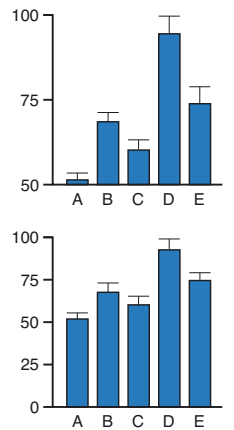

Y-axis of bar charts has to start at zero. Bar height represents discrete value; a non-zero start could be misleading.
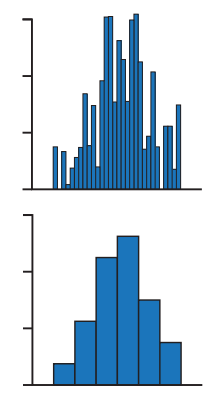

Group data into bins to increase clarity of the trend and reduce the effects of outliers.
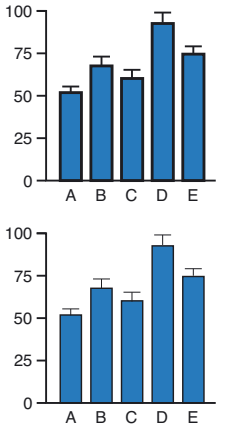

Be deliberate

about width of

outlines of bars:

ideally $75 \%$ of the

weight of the

axes.
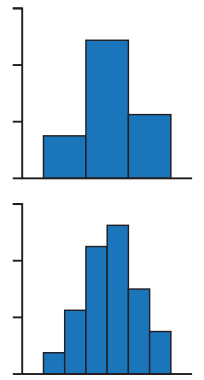

Have at least five bins. This allows meaningful conclusions about a dataset.
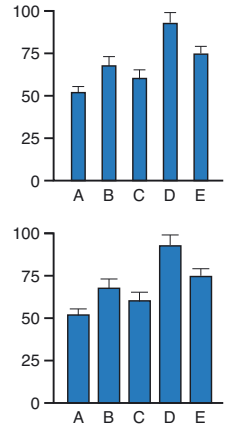

Width of bars and spacing: not too flimsy, not too thick. Spacing: ideally, about a third of the bar width.
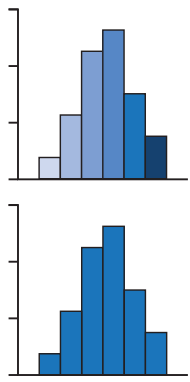

If the histogram represents a single dataset, use the same color for all bins.
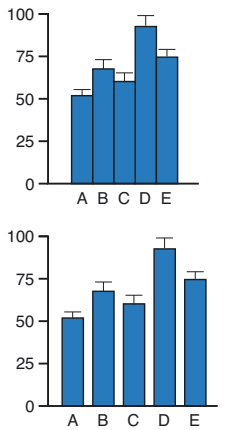

Don't place

individual bars

next to each

other. The bars

need some

space between

themselves.
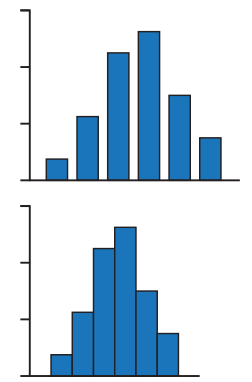

Don't use spacing

between bars on a histogram as you would on a bar chart.
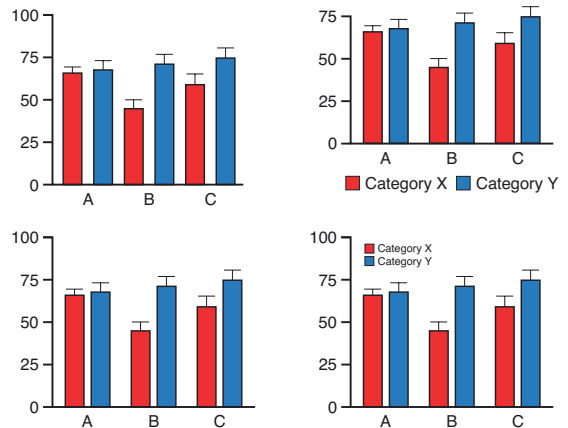

For groups of data, place a larger space between the different variables on the $x$-axis than between the different bars. Keep the order of the bars consistent.

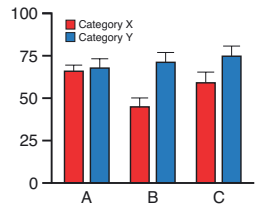

Place keys to data categories to the right or on the top of a bar graph. Best way to save space: place the key within the area of the bar graph itself.
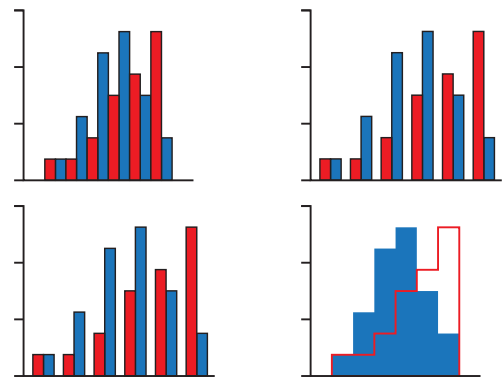

Use spacing between bars if there are two or more datasets plotted on the same histogram.
Often, a better solution is to overlay one of the histograms with its outline only.
Figure B.27: Advice for bar charts and histograms (reproduced from [Car12] with permission).
[Car12]. Moreover, you will see how to apply the principles of organizing information (cf. Sect. B.2).

Often, the properties of entities have different scales and units. Readers usually expect that rows correspond to entities, and columns correspond to properties. This organization makes it easier to compare entities in terms of individual properties by focusing on the values in particular columns. It is easy to spot exceptional cases if they are printed vertically and the eye can move downwards. The example in Table B.1 shows this effect with two tables that contain the same data.

Follow the Gestalt principle of Hierarchy when you display information that can be grouped. Often, there is more than one way how to group data. Make a conscious decision about how you organize the table. Different forms of organization emphasize different aspects. For instance, the table to the left in Table B.2 emphasizes the comparison between men and women. In contrast, the table to the right highlights the differences between the years. 
Table B.1: The table to the left is easier to read than the table to the right (reproduced from [Cari2] with permission).

\begin{tabular}{lccr}
\hline Lake & Area $\left(\mathrm{km}^{2}\right)$ & Length $(\mathrm{km})$ & Depth $(\mathrm{m})$ \\
\hline Malawi & 30,044 & 579 & 706 \\
Tanganyika & 32,893 & 676 & 1470 \\
Victoria & 59,485 & 322 & 84 \\
\hline
\end{tabular}

\begin{tabular}{lccr}
\hline Lake & Malawi & Tanganyika & Victoria \\
\hline Area $\left(\mathrm{km}^{2}\right)$ & 30,044 & 32,893 & 59,485 \\
Length $(\mathrm{km})$ & 579 & 676 & 322 \\
Depth $(\mathrm{m})$ & 706 & 1470 & 84 \\
\hline
\end{tabular}

Table B.2: Number of men and women selected by NASA to be astronauts by year of selection (reproduced from [Car12] with permission).

\begin{tabular}{lcccccccc}
\hline & \multicolumn{3}{c}{ Men } & & \multicolumn{3}{c}{ Women } \\
\cline { 2 - 4 } \cline { 7 - 9 } & $\mathbf{1 9 8 0}$ & $\mathbf{1 9 9 0}$ & $\mathbf{2 0 0 0}$ & & $\mathbf{1 9 8 0}$ & $\mathbf{1 9 9 0}$ & $\mathbf{2 0 0 0}$ \\
\hline Mission specialist & 9 & 12 & 7 & & 2 & 4 & 3 \\
Pilot & 8 & 6 & 7 & & 0 & 1 & 0 \\
\hline Total & 17 & 18 & 14 & & 2 & 5 & 3 \\
\hline
\end{tabular}

\begin{tabular}{|c|c|c|c|c|c|c|}
\hline & \multicolumn{2}{|c|}{1980} & \multicolumn{2}{|c|}{1990} & \multicolumn{2}{|c|}{2000} \\
\hline & $\mathbf{M}$ & $\mathbf{F}$ & $\mathbf{M}$ & $\mathbf{F}$ & $\mathbf{M}$ & $\mathbf{F}$ \\
\hline Mission specialist & 9 & 2 & 12 & 4 & 7 & 3 \\
\hline Pilot & 8 & 0 & 6 & 1 & 7 & 0 \\
\hline Total & 17 & 2 & 18 & 5 & 14 & 3 \\
\hline
\end{tabular}

Make a conscious decision on how to order the columns. Revisit the five hat racks design principle in Sect. B.2.3. If there is no logical ordering, e. g., from very generic properties to more specific ones, order the columns alphabetically. Usually, derived pieces of information, results, and insights are to the right of labels and descriptive pieces of information.

Choosing an adequate order also applies to rows. Instead of sorting the rows alphabetically based on their label in the first column, consider sorting them based on a particular column's value. The resulting order can improve legibility a lot (principle of continuity). Note that sorting the table by a specific column puts some emphasis on that particular column. This principle is visualized in Table B.3.

Table B.3: Listing planets in order from the sun, in alphabetical order, and in descending order of diameter (reproduced from [Car12] with permission).

\begin{tabular}{lrr}
\hline Planet & Diameter & Mass \\
\hline Mercury & 0.38 & 0.06 \\
Venus & 0.95 & 0.82 \\
Earth & 1.00 & 1.00 \\
Mars & 0.53 & 0.11 \\
Jupiter & 11.21 & 317.80 \\
Saturn & 9.45 & 95.20 \\
Uranus & 4.01 & 14.60 \\
Neptune & 3.88 & 17.20 \\
\hline
\end{tabular}

\begin{tabular}{lrr}
\hline Planet & Diameter & Mass \\
\hline Earth & 1.00 & 1.00 \\
Jupiter & 11.21 & 317.80 \\
Mars & 0.53 & 0.11 \\
Mercury & 0.38 & 0.06 \\
Neptune & 3.88 & 17.20 \\
Saturn & 9.45 & 95.20 \\
Uranus & 4.01 & 14.60 \\
Venus & 0.95 & 0.82 \\
\hline
\end{tabular}

\begin{tabular}{lrr}
\hline Planet & Diameter & Mass \\
\hline Jupiter & 11.21 & 317.80 \\
Saturn & 9.45 & 95.20 \\
Uranus & 4.01 & 14.60 \\
Neptune & 3.88 & 17.20 \\
Earth & 1.00 & 1.00 \\
Venus & 0.95 & 0.82 \\
Mars & 0.53 & 0.11 \\
Mercury & 0.38 & 0.06 \\
\hline
\end{tabular}





\section{C | About the Template}

This appendix provides additional information about less-often needed features of the template. Moreover, it contains a brief overview of the template's history.

\section{C.1 Further Template Features}

This section explains customization options and technical details. For a thesis at the PSI Chair, you should stick with the defaults.

\section{C.1.1 Printing Format}

This thesis template is designed for double-sided printing (i. e., content on the front and back of pages) as most theses are printed and bound this way. ${ }^{1}$ Switching to one-sided printing is as simple as uncommenting the oneside option of the documentclass command at the top of the main.tex file. You may then wish to adjust the margins to suit specifications from your institution.

The headers for the pages contain the page number on the outer side (so it is easy to flick through to the page you want) and the chapter name on the inner side.

The font size is set to 11 points by default with single line spacing; again, you can tune the text size and spacing using the options at the top of main. tex. The spacing can be influenced by replacing the singlespacing with onehalfspacing or doublespacing.

\section{C.1.2 Using US Letter Paper}

The paper size used in the template is A4, which is the standard size in Europe. If you are using this thesis template elsewhere, for instance, in the United States, then you may have to change the A4 paper size to the US Letter size.

Due to the differences in the paper size, the resulting margins may differ from what you like or require. You may need to adapt the page geometry settings in setup. tex in this case. 1: At the PSI Chair, we highly encourage you to
use double-sided printing. 


\section{C.1.3 References}

The template uses biblatex to format the bibliography and references such as this one [Mur+10]. The template uses a citation style that creates in-text citations with the author(s) initials and the year of the publication. Multiple references are separated by semicolons (e. g., [Bon+14; Sol17]). To see how you use references, look at the source files of this guide. If you choose a suitable BibTeX reference manager, you can copy and paste or drag and drop references into the document.

The bibliography is typeset with references listed in alphabetical order by the first author's last name. To see how LaTeX typesets the bibliography, look at the end of this document (or just click on the reference links in in-text citations).

BibTeX Backend As the "old" bibtex backend does not correctly handle Unicode character encoding (i. e., "international" characters), we use the more modern biber BibTeX engine in this template.

Here, we cite a lot of references so that the list of references gets populated [Aca+14; AM14; BFKo1; Bon+14; Cha81; DMSo4; DRo8; Fer+16; FHF13; Goo12; GY19; HAF12; HBo6; HBF13; Her+12; Her+14; Her14; Hino3; HWFo9; KAo3; Köpo6; LAo9; LMD10; Mur+10; Orto6; Rayo1; Sol17; VGMo3; WG13; WHFo7; Yan1o; ZX14].

\section{C.2 Contributors and History}

This guide has been written by Dominik Herrmann. The LaTeX template has been created by Dominik Herrmann with support by Fabian Lamprecht. Dominik and Fabian are affiliated with the Privacy and Security in Information Systems Group at University of Bamberg (https: //www.uni-bamberg.de/psi/).

The PSI Template has its own document class, PSIThesis.cls. It has been derived from MastersDoctoralThesis.cls (https://www.latextemplates. com/template/masters-doctoral-thesis).

The MastersDoctoralThesis LaTeX thesis template is based initially on a LaTeX style file created by Steve R. Gunn from the University of Southampton (UK), department of Electronics and Computer Science. You can find his original thesis style file at his site at http://www.ecs.soton.ac.uk/ sr $\mathrm{g} /$ softwaretools/document/templates/ (link not available as of 2019).

Steve's ecsthesis.cls was then taken by Sunil Patel, who modified it by creating a skeleton framework and folder structure for a thesis. The resulting template is available on Sunil's site at http://www.sunilpatel.co .uk/thesis-template.

Sunil's template was made available through http://www.LaTeXTemp lates.com where it was modified many times based on user requests and questions. Version 2.0 and onwards of this template represents a 
significant modification to Sunil's template and is, in fact, hardly recognizable. The work to make version 2.0 possible was carried out by Vel and Johannes Böttcher.

\section{C.3 License}

We have chosen a license that allows you to use the template for your thesis and make changes as needed. You are not required to publish your thesis or its source code under the same license. If you fork the template or the guide, however, you have to comply with the following licensing restrictions.

This guide and the template are made available under the Creative Commons license CC BY-SA 4.0 (http://creativecommons.org/licenses/by-sa /4.0/) with two exceptions:

1. Some excerpts, figures, and tables in Chapter 2 and Appendix B have been taken from the literature. The respective elements are explicitly marked with a citation and a note regarding the permission to re-use. They are not covered by the CC license. Permission to re-use and distribute these figures, tables, and excerpts must be obtained from the respective copyright holders.

2. Parts of Chapter 1 and Appendix $C$ contain content from the MastersDoctoralThesis template mentioned above, which is licensed under CC BY-SA 3.o (http://creativecommons.org/licenses/by-nc-sa/3.0/). The original content has been written by Sunil Patel (www.sunilpatel.co.uk) and Vel (LaTeXTemplates.com).

As this guide contains copyrighted material from third parties, you cannot host your own copy of this guide online. Please use the URLs printed on the title page to link to this document.

The files PSIThesis.cls, setup.tex, and titlepage. tex are made available under the LPPL v1.3c (http://www.latex-project.org/lppl).

The fonts that are included in this template in the fonts directory are licensed under the Apache License 2.o (Roboto sans-serif font) and the SIL OFL Version 1.1 (Iosevka monospace font). 



\section{References}

[Aca+14] G Acar et al. The Web Never Forgets: Persistent Tracking Mechanisms in the Wild. In: CCS 2014. ACM, 2014, pp. 674-689 (page 44).

[AM14] Anderson, Ross and Murdoch, Steven J. EMV: why payment systems fail. In: Communications of the ACM 57(6):(June 2014), 24-28. http: //dl.acm.org/citation.cfm?doid=2602695.2602321 (pages 8, 9, 44).

[BFKo1] O Berthold, H Federrath, and S Köpsell. Web MIXes: A System for Anonymous and Unobservable Internet Access. In: International Workshop on Design Issues in Anonymity and Unobservability. Vol. 2009. LNCS. Springer, 2001, pp. 115-129 (page 44).

[Bon+14] M Bond et al. Chip and Skim: Cloning EMV Cards with the Pre-play Attack. In: 2014 IEEE Symposium on Security and Privacy. May 2014, pp. 4964 (page 44).

[Buc+o8] E Buchanan et al. When good instructions go bad: generalizing return-oriented programming to RISC. In: Proceedings of the 2008 ACM Conference on Computer and Communications Security, CCS 2008, Alexandria, Virginia, USA, October 27-31, 2008. Ed. by P Ning, PF Syverson, and S Jha. ACM, 2008, pp. 27-38. https://doi.org/10.1145/1455770.1455776 (page 9).

[Car12] M Carter. Designing Science Presentations: A Visual Guide to Figures, Papers, Slides, Posters, and More. USA: Academic Press, Inc., 2012 (pages 27, 29, 30, 33, 35-37, 39-41).

[Cha81] D Chaum. Untraceable electronic mail, return addresses, and digital pseudonyms. In: Communications of the ACM 24(2):(1981) (page 44).

[DRo8] T Dierks and E Rescorla. The Transport Layer Security (TLS) Protocol Version 1.2. RFC 5246. RFC Editor, Aug. 2008 (page 44).

[Die+18] C Dietrich et al. Investigating System Operators' Perspective on Security Misconfigurations. In: Conference on Computer and Communications Security (CCS). ACM, 2018, pp. 1272-1289 (page 9).
[DMSo4] R Dingledine, N Mathewson, and PF Syverson. Tor: The Second-Generation Onion Router. In: 13th USENIX Security Symposium. USENIX, 2004, pp. 303-320 (page 44).

[Fer+16] H Ferradi et al. When Organized Crime Applies Academic Results: A Forensic Analysis of an in-Card Listening Device. In: Journal of Cryptographic Engineering 6(1):(Apr. 2016), 49-59 (page 44).

[FHF13] KP Fuchs, D Herrmann, and H Federrath. Generating Realistic Application Workloads for Mix-Based Systems for Controllable, Repeatable and Usable Experimentation. In: IFIP SEC 2013. Vol. 405. AICT. Springer, 2013, pp. 162-175 (page 44).

[GY19] LA Galloway and T Yunusov. Visa card vulnerability can bypass contactless limits. Accessed: 2019-0813. July 29, 2019. https://www.ptsecurity.com/wwen/about/news/visa-card-vulnerability-can-bypasscontactless-limits/ (visited on o8/13/2019) (page 44).

[Goo12] S Goodson. If You're Not Paying For It, You Become The Product. Forbes.com, http:// onforb. es/wVrU4G (visited on 6 Jan 2015). 2012 (page 44).

[HBo6] E Haselsteiner and K Breitfuß. Security in Near Field Communication (NFC). In:(Jan. 2006), 11 (page 44).

[Her14] D Herrmann. Beobachtungsmöglichkeiten im Domain Name System: Angriffe auf die Privatsphäre und Techniken zum Selbstdatenschutz. PhD thesis. Universität Hamburg, 2014 (page 44).

[HAF12] D Herrmann, C Arndt, and H Federrath. IPv6 Prefix Alteration: An Opportunity to Improve Online Privacy. In: 1st Workshop on Privacy and Data Protection Technology, co-located with Amsterdam Privacy Conference. 2012 (page 44).

[HBF13] D Herrmann, C Banse, and H Federrath. Behavior-based Tracking: Exploiting Characteristic Patterns in DNS Traffic. In: Computers es Security 39A:(Nov. 2013), 17-33 (page 44). 
[HWFo9] D Herrmann, R Wendolsky, and H Federrath. Website Fingerprinting: Attacking Popular Privacy Enhancing Technologies with the Multinomial Näive-Bayes Classifier. In: CCSW 2009. ACM, 2009, pp. 31-42 (page 44).

[Her+12] D Herrmann et al. Analyzing Characteristic Host Access Patterns for Re-identification of Web User Sessions. In: NordSec 2010. Vol. 7127. LNCS. Springer, 2012, pp. 136-154 (page 44).

[Her+14] D Herrmann et al. EncDNS: A Lightweight Privacy-Preserving Name Resolution Service. In: ESORICS 2014. Vol. 8712. LNCS. Springer, 2014, pp. 37-55 (page 44).

[Hino3] A Hintz. Fingerprinting Websites Using Traffic Analysis. In: PET Workshop 2002. Vol. 2482. LNCS. Springer, 2003, pp. 171-178 (pages 6, 8, 44).

[Köpo6] S Köpsell. Low Latency Anonymous Communication - How Long Are Users Willing to Wait? In: ETRICS 2006. Vol. 3995. LNCS. Springer, 2006, pp. 221-237 (page 44).

[KAo3] Kou, Weidong and Agnew, Gordon. Secure Electronic Transactions: Overview, Capabilities, and Current Status. In: Payment Technologies for E-Commerce. Berlin, Heidelberg: Springer Berlin Heidelberg, 2003, pp. 211-226. http://www.springerlink. com/index/10.1007/978-3-662-05322-5_10 (pages 8, 9, 44).

[LAo9] N Laleh and M Abdollahi Azgomi. A Taxonomy of Frauds and Fraud Detection Techniques. In: Information Systems, Technology and Management. Ed. by SK Prasad et al. Communications in Computer and Information Science. Springer Berlin Heidelberg, 2009, pp. 256-267 (page 44).

[Lid+10] W Lidwell et al. Universal Principles of Design, Revised and Updated: 125 Ways to Enhance Usability, Influence Perception, Increase Appeal, Make Better Design Decisions, and Teach Through Design. 2nd ed. Rockport Publishers, 2010 (pages 27, 30-32).

[LMD10] K Loesing, SJ Murdoch, and R Dingledine. A Case Study on Measuring Statistical Data in the Tor Anonymity Network. In: FC 2010 Workshops. Vol. 6054. LNCS. Springer, 2010, pp. 203-215 (page 44).

[Mur+10] Murdoch, Steven J. et al. Chip and PIN is Broken. In: 2010 IEEE Symposium on Security and
Privacy. Oakland, CA, USA: IEEE, 2010, pp. 433-446. http://ieeexplore.ieee.org/document/5504801/ (pages 8, 9, 44).

[Orto6] Ortiz, S. Is Near-Field Communication Close to Success? In: Computer 39(3):(Mar. 2006), 18-20. http://ieeexplore.ieee.org/document/1607943/ (page 44).

[Rayo1] JF Raymond. Traffic Analysis: Protocols, Attacks, Design Issues, and Open Problems. In: International Workshop on Design Issues in Anonymity and Unobservability. Vol. 2009. LNCS. Springer, 2001, pp. 10-29 (page 44).

[Sol17] S Solat. Security of Electronic Payment Systems: A Comprehensive Survey. In: arXiv:1701.04556 [cs]:(Jan. 2017). arXiv: 1701.04556. http : / / arxiv . org/abs/1701.04556 (page 44).

[VGMo3] T Vila, R Greenstadt, and D Molnar. Why we can't be bothered to read privacy policies: models of privacy economics as a lemons market. In: ICEC 2003. ACM, 2003, pp. 403-407 (page 44).

[WG13] T Wang and I Goldberg. Improved website fingerprinting on Tor. In: WPES 2013. ACM, 2013, pp. 201-212 (page 44).

[War12] C Ware. Information Visualization: Perception for Design. 3rd ed. San Francisco, CA, USA: Morgan Kaufmann Publishers Inc., 2012 (pages 27-30, 32-34, 37).

[WHFo7] R Wendolsky, D Herrmann, and H Federrath. Performance Comparison of Low-Latency Anonymisation Services from a User Perspective. In: PET Workshop 2007. Vol. 4776. LNCS. Springer, 2007, pp. 233-253 (page 44).

[Yan1o] Y Yang. Web user behavioral profiling for user identification. In: Decision Support Systems 49:(2010), 261-271 (page 44).

[ZX14] R Zhou and G Xing. nShield: a noninvasive NFC security system for mobiledevices. In: Proceedings of the 12th annual international conference on Mobile systems, applications, and services - MobiSys '14. the 12th annual international conference. Bretton Woods, New Hampshire, USA: ACM Press, 2014, pp. 95-108. http://dl.acm.org/citation.cfm? doid= 2594368.2594376 (visited on 08/18/2019) (page 44). 


\section{Declaration of Authorship}

Ich erkläre hiermit gemäß $\$ 17$ Abs. 2 APO, dass ich die vorstehende Bachelorarbeit selbständig verfasst und keine anderen als die angegebenen Quellen und Hilfsmittel benutzt habe.

Bamberg, den

Dominik Herrmann 